\title{
SEARCH FOR EARLY GAMMA-RAY PRODUCTION IN SUPERNOVAE LOCATED IN A DENSE CIRCUMSTELLAR MEDIUM WITH THE FERMI LAT
}

M. Ackermann ${ }^{1}$, I. Arcavi ${ }^{57,58}$, L. Baldini ${ }^{2}$, J. Ballet ${ }^{3}$, G. Barbiellini ${ }^{4,5}$, D. Bastieri ${ }^{6,7}$, R. Bellazzini ${ }^{8}$, E. Bissaldi ${ }^{9}$, R. D. Blandford ${ }^{10}$, R. Bonino ${ }^{11,12}$, E. Bottacini ${ }^{10}$, T. J. BrandT ${ }^{13}$, J. Bregeon ${ }^{14}$, P. Bruel ${ }^{15}$, R. Buehler ${ }^{1}$, S. Buson ${ }^{6,7}$, G. A. Caliandro ${ }^{10,16}$, R. A. Cameron ${ }^{10}$, M. Caragiulo ${ }^{9}$, P. A. Caraveo ${ }^{17}$, E. Cavazzuti $^{18}$, C. Cecchi ${ }^{19,20}$, E. Charles $^{10}$, A. CheKhtman ${ }^{21}$, J. Chiang ${ }^{10}$, G. Chiaro ${ }^{7}$, S. Ciprini ${ }^{18,19,22}$, R. Claus ${ }^{10}$, J. Cohen-Tanugi ${ }^{14}$, S. Cutini ${ }^{18,19,22}$, F. D’Ammando ${ }^{23,24}$, A. de Angelis ${ }^{25}$, F. De Palma ${ }^{9,26}$, R. Desiante ${ }^{4,27}$, L. Di Venere ${ }^{28}$, P. S. Drell ${ }^{10}$, C. Favuzzi $^{9,28}$, S. J. Fegan ${ }^{15}$, A. FranckowiaK ${ }^{10}$, S. FunK ${ }^{10}$, P. Fusco ${ }^{9,28}$, A. Gal-Yam ${ }^{56}$, F. Gargano ${ }^{9}$, D. Gasparrini ${ }^{18,19,22}$, N. Giglietto 9,28 , F. Giordano, ${ }^{9}$, M. Giroletti $^{23}$, T. Glanzman ${ }^{10}$, G. Godfrey ${ }^{10}$, I. A. Grenier ${ }^{3}$, J. E. Grove ${ }^{29}$,

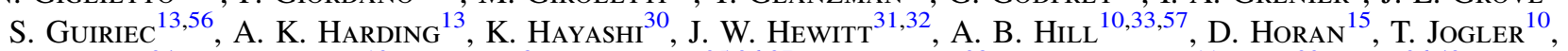

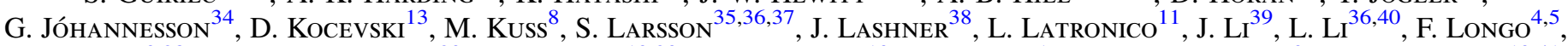
F. Loparco ${ }^{9,28}$, M. N. Lovellette ${ }^{29}$, P. Lubrano ${ }^{19,20}$, D. Malyshev ${ }^{10}$, M. MaYer ${ }^{1}$, M. N. Mazziotta ${ }^{9}$, J. E. McEnery ${ }^{13,41}$, P. F. Michelson ${ }^{10}$, T. Mizuno ${ }^{42}$, M. E. Monzani ${ }^{10}$, A. Morselli ${ }^{43}$, K. Murase ${ }^{44,45}$, P. Nugent ${ }^{46,47}$, E. Nuss ${ }^{14}$, E. OfeK ${ }^{48}$, T. Ohsugi ${ }^{42}$, M. Orienti ${ }^{23}$, E. Orlando ${ }^{10}$, J. F. Ormes ${ }^{49}$, D. Paneque ${ }^{10,50}$, M. Pesce-Rollins ${ }^{8}$, F. Piron ${ }^{14}$, G. Pivato ${ }^{8}$,

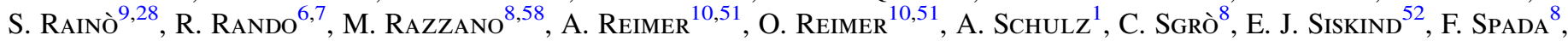

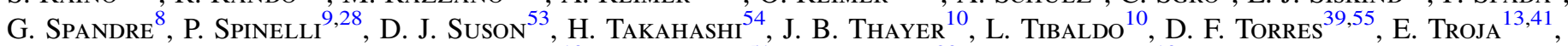

G. VIANELlo ${ }^{10}$, M. Werner ${ }^{51}$, K. S. Wood ${ }^{29}$, AND M. Wood ${ }^{10}$

${ }^{1}$ Deutsches Elektronen Synchrotron DESY, D-15738 Zeuthen, Germany

${ }^{2}$ Università di Pisa and Istituto Nazionale di Fisica Nucleare, Sezione di Pisa I-56127 Pisa, Italy

${ }^{3}$ Laboratoire AIM, CEA-IRFU/CNRS/Université Paris Diderot, Service d'Astrophysique, CEA Saclay, F-91191 Gif sur Yvette, France

${ }^{4}$ Istituto Nazionale di Fisica Nucleare, Sezione di Trieste, I-34127 Trieste, Italy

${ }^{5}$ Dipartimento di Fisica, Università di Trieste, I-34127 Trieste, Italy

${ }^{6}$ Istituto Nazionale di Fisica Nucleare, Sezione di Padova, I-35131 Padova, Italy

${ }^{7}$ Dipartimento di Fisica e Astronomia "G. Galilei," Università di Padova, I-35131 Padova, Italy

${ }_{9}^{8}$ Istituto Nazionale di Fisica Nucleare, Sezione di Pisa, I-56127 Pisa, Italy

${ }^{9}$ Istituto Nazionale di Fisica Nucleare, Sezione di Bari, I-70126 Bari, Italy

${ }^{10}$ W. W. Hansen Experimental Physics Laboratory, Kavli Institute for Particle Astrophysics and Cosmology, Department of Physics and SLAC National Accelerator

Laboratory, Stanford University, Stanford, CA 94305, USA; afrancko@slac.stanford.edu

${ }^{11}$ Istituto Nazionale di Fisica Nucleare, Sezione di Torino, I-10125 Torino, Italy

${ }^{12}$ Dipartimento di Fisica Generale "Amadeo Avogadro," Università degli Studi di Torino, I-10125 Torino, Italy

${ }^{13}$ NASA Goddard Space Flight Center, Greenbelt, MD 20771, USA

${ }^{14}$ Laboratoire Univers et Particules de Montpellier, Université Montpellier, CNRS/IN2P3, Montpellier, France

${ }^{15}$ Laboratoire Leprince-Ringuet, École polytechnique, CNRS/IN2P3, Palaiseau, France

${ }_{17}$ Consorzio Interuniversitario per la Fisica Spaziale (CIFS), I-10133 Torino, Italy

${ }_{18}^{7}$ INAF-Istituto di Astrofisica Spaziale e Fisica Cosmica, I-20133 Milano, Italy

${ }_{18}$ Agenzia Spaziale Italiana (ASI) Science Data Center, I-00133 Roma, Italy

${ }^{19}$ Istituto Nazionale di Fisica Nucleare, Sezione di Perugia, I-06123 Perugia, Italy

${ }^{20}$ Dipartimento di Fisica, Università degli Studi di Perugia, I-06123 Perugia, Italy

${ }^{21}$ College of Science, George Mason University, Fairfax, VA 22030, resident at Naval Research Laboratory, Washington, DC 20375, USA

22 INAF Osservatorio Astronomico di Roma, I-00040 Monte Porzio Catone (Roma), Italy

${ }^{23}$ INAF Istituto di Radioastronomia, I-40129 Bologna, Italy

${ }^{24}$ Dipartimento di Astronomia, Università di Bologna, I-40127 Bologna, Italy

${ }^{25}$ Dipartimento di Fisica, Università di Udine and Istituto Nazionale di Fisica Nucleare, Sezione di Trieste, Gruppo Collegato di Udine, I-33100 Udine, Italy

${ }^{26}$ Università Telematica Pegaso, Piazza Trieste e Trento, 48, I-80132 Napoli, Italy

${ }^{27}$ Università di Udine, I-33100 Udine, Italy

${ }^{28}$ Dipartimento di Fisica "M. Merlin" dell’Università e del Politecnico di Bari, I-70126 Bari, Italy

${ }^{29}$ Space Science Division, Naval Research Laboratory, Washington, DC 20375-5352, USA

${ }^{30}$ Institute of Space and Astronautical Science, Japan Aerospace Exploration Agency, 3-1-1 Yoshinodai, Chuo-ku, Sagamihara, Kanagawa 252-5210, Japan

${ }_{31}$ Department of Physics and Center for Space Sciences and Technology, University of Maryland Baltimore County, Baltimore, MD 21250, USA

${ }^{32}$ Center for Research and Exploration in Space Science and Technology (CRESST) and NASA Goddard Space Flight Center, Greenbelt, MD 20771, USA

${ }^{33}$ School of Physics and Astronomy, University of Southampton, Highfield, Southampton, SO17 1BJ, UK

${ }^{34}$ Science Institute, University of Iceland, IS-107 Reykjavik, Iceland

${ }^{35}$ Department of Physics, Stockholm University, AlbaNova, SE-106 91 Stockholm, Sweden

${ }^{36}$ The Oskar Klein Centre for Cosmoparticle Physics, AlbaNova, SE-106 91 Stockholm, Sweden

37 Department of Astronomy, Stockholm University, SE-106 91 Stockholm, Sweden

${ }^{38}$ Wesleyan University, 45 Wyllys Avenue, Middletown, CT 06459, USA

${ }^{39}$ Institute of Space Sciences (IEEC-CSIC), Campus UAB, E-08193 Barcelona, Spain

${ }^{40}$ Department of Physics, KTH Royal Institute of Technology, AlbaNova, SE-106 91 Stockholm, Sweden

${ }_{41}^{41}$ Department of Physics and Department of Astronomy, University of Maryland, College Park, MD 20742, USA

${ }^{42}$ Hiroshima Astrophysical Science Center, Hiroshima University, Higashi-Hiroshima, Hiroshima 739-8526, Japan

${ }^{43}$ Istituto Nazionale di Fisica Nucleare, Sezione di Roma “Tor Vergata," I-00133 Roma, Italy

${ }^{44}$ Institute for Advanced Study, Princeton, NJ 08540, USA

${ }^{45}$ Center for Particle and Gravitational Astrophysics, Department of Physics, Department of Astronomy and Astrophysics,

The Pennsylvania State University, University Park, Pennsylvania, 16802, USA

${ }^{46}$ Lawrence Berkeley National Lab, 1 Cyclotron Road, Berkeley, CA 94720, USA

${ }^{47}$ Department of Astronomy, University of California, Berkeley, CA 94720-3411, USA

${ }^{48}$ Benoziyo Center for Astrophysics, Weizmann Institute of Science, 76100 Rehovot, Israel 


$$
\begin{gathered}
{ }^{49} \text { Department of Physics and Astronomy, University of Denver, Denver, CO 80208, USA } \\
50 \text { Max-Planck-Institut für Physik, D-80805 München, Germany } \\
51 \text { Institut für Astro- und Teilchenphysik and Institut für Theoretische Physik, Leopold-Franzens-Universität Innsbruck, A-6020 Innsbruck, Austria } \\
52 \text { NYCB Real-Time Computing Inc., Lattingtown, NY 11560-1025, USA } \\
53 \text { Department of Chemistry and Physics, Purdue University Calumet, Hammond, IN 46323-2094, USA } \\
{ }^{54} \text { Department of Physical Sciences, Hiroshima University, Higashi-Hiroshima, Hiroshima 739-8526, Japan } \\
55 \text { Institució Catalana de Recerca i Estudis Avançats (ICREA), Barcelona, Spain } \\
56 \text { Department of Particle Physics and Astrophysics, Weizmann Institute of Science, Rehovot 76100, Israel } \\
57 \text { Las Cumbres Observatory Global Telescope Network, 6740 Cortona Dr., Suite 102, Goleta, CA 93117, USA } \\
58 \text { Kavli Institute for Theoretical Physics, University of California, Santa Barbara, CA 93106, USA } \\
\text { Received 2015 April 10; accepted 2015 June 2; published 2015 July 9 }
\end{gathered}
$$

\begin{abstract}
Supernovae ( $\mathrm{SNe}$ ) exploding in a dense circumstellar medium (CSM) are hypothesized to accelerate cosmic rays in collisionless shocks and emit $\mathrm{GeV} \gamma$-rays and $\mathrm{TeV}$ neutrinos on a timescale of several months. We perform the first systematic search for $\gamma$-ray emission in Fermi Large Area Telescope data in the energy range from $100 \mathrm{MeV}$ to $300 \mathrm{GeV}$ from the ensemble of $147 \mathrm{SNe}$ Type IIn exploding in a dense CSM. We search for a $\gamma$-ray excess at each $\mathrm{SNe}$ location in a one-year time window. In order to enhance a possible weak signal, we simultaneously study the closest and optically brightest sources of our sample in a joint-likelihood analysis in three different time windows (1 year, 6 months, and 3 months). For the most promising source of the sample, SN 2010jl (PTF 10aaxf), we repeat the analysis with an extended time window lasting 4.5 years. We do not find a significant excess in $\gamma$-rays for any individual source nor for the combined sources and provide model-independent flux upper limits for both cases. In addition, we derive limits on the $\gamma$-ray luminosity and the ratio of $\gamma$-ray-to-optical luminosity ratio as a function of the index of the proton injection spectrum assuming a generic $\gamma$-ray production model. Furthermore, we present detailed flux predictions based on multi-wavelength observations and the corresponding flux upper limit at a $95 \%$ confidence level (CL) for the source SN 2010jl (PTF 10aaxf).
\end{abstract}

Key words: cosmic rays - gamma rays: general - methods: data analysis - supernovae: general

\section{INTRODUCTION}

The Large Area Telescope (LAT) on board the Fermi Gamma-ray Space Telescope mission unanticipatedly detected $\gamma$-ray emission from five Galactic novae (Abdo et al. 2010; Cheung et al. 2013; Hays et al. 2013; Hill et al. 2013). The origin of the $\gamma$-ray emission is still unclear. Shocks produced by an expansion of the nova shell into the wind provided by the companion star or internal shocks within the ejecta might be responsible for the acceleration of particles to relativistic energies and ensuing high-energy $\gamma$-ray emission. A similar mechanism but with much larger energy output is hypothesized to produce $\gamma$-rays in supernovae ( $\mathrm{SNe}$ ), yielding potentially detectable $\gamma$-ray emission even from extragalactic sources. Murase et al. (2011, 2014) and Katz et al. (2011) showed that if the SN progenitor is surrounded by an optically thick circumstellar medium (CSM), then a collisionless shock is necessarily formed after the shock breakout. The collisionless shock may accelerate protons and electrons to high energies, which emit photons from the radio-submillimeter through $\mathrm{GeV}$ energies and $\mathrm{TeV}$ neutrinos. Such conditions appear in shocks propagating through dense circumstellar matter (e.g., wind). Recently several candidates for such $\mathrm{SNe}$ powered by interactions with a dense CSM were found (e.g., Ofek et al. 2007, 2014b; Smith et al. 2009; Zhang et al. 2012) and some superluminous $\mathrm{SNe}$ were suggested to be powered by interactions (e.g., Chevalier \& Irwin 2011; Quimby et al. 2011). Such interaction-powered SNe may also be Pevatrons, implying their importance for the origin of the knee structure in the cosmic-ray spectrum (Sveshnikova 2003; Murase

\footnotetext{
59 NASA Postdoctoral Program Fellow, USA.

60 Funded by a Marie Curie IOF, FP7/2007-2013-Grant agreement no. 275861.

${ }^{61}$ Funded by contract FIRB-2012-RBFR12PM1F from the Italian Ministry of Education, University and Research (MIUR).
}

et al. 2014). Both $\gamma$-rays and neutrinos originate from pp and $\mathrm{p} \gamma$ interactions producing pions, which in the neutral case decay to $\gamma$-rays and in the charged case produce neutrinos in the decay chain. Thus, the initial neutrino and $\gamma$-ray spectra have the same shape. Contrary to neutrinos, $\gamma$-rays might be affected by absorption in the CSM and/or two-photon annihilation with low-energy photons produced at the forward shock (Murase et al. 2011). However, arguments made in Murase et al. (2014) suggest that $\mathrm{GeV} \gamma$-rays can escape the system without severe attenuation if the shock velocity is in the right range, especially late after the shock breakout.

Motivated by the fact that the LAT has detected $\gamma$-ray emission from novae, we are presenting the first systematic search for $\gamma$-ray emission from SNe IIn in Fermi LAT data from $100 \mathrm{MeV}$ to $300 \mathrm{GeV}$. Considering current theoretical uncertainties we are aiming for a model-independent search. SNe positions and explosion times are given by optical surveys such as the Palomar Transient Factory (PTF; Law et al. 2009; Rau et al. 2009).

We present the sample of SNe used in the $\gamma$-ray data analysis in Section 2. Section 3 describes the Fermi LAT data analysis followed by an interpretation of our results in Section 4, and conclusions in Section 5.

\section{SNE SAMPLE}

$\mathrm{SNe}$ IIn and Ibn are the best candidates to be found interacting with a dense CSM. Their long-lasting bright optical light curves are believed to be powered by the interaction of the ejecta with a massive CSM (Svirski et al. 2012). SNe of these types are often accompanied by precursor mass-ejection events (Ofek et al. 2014a). Here we mainly use the PTF SN sample along with publicly available SNe IIn discovered since the launch of Fermi in 2008. Appendix A lists all of the $147 \mathrm{SNe}$ of this sample that we consider in our $\gamma$-ray search, i.e., all sources 


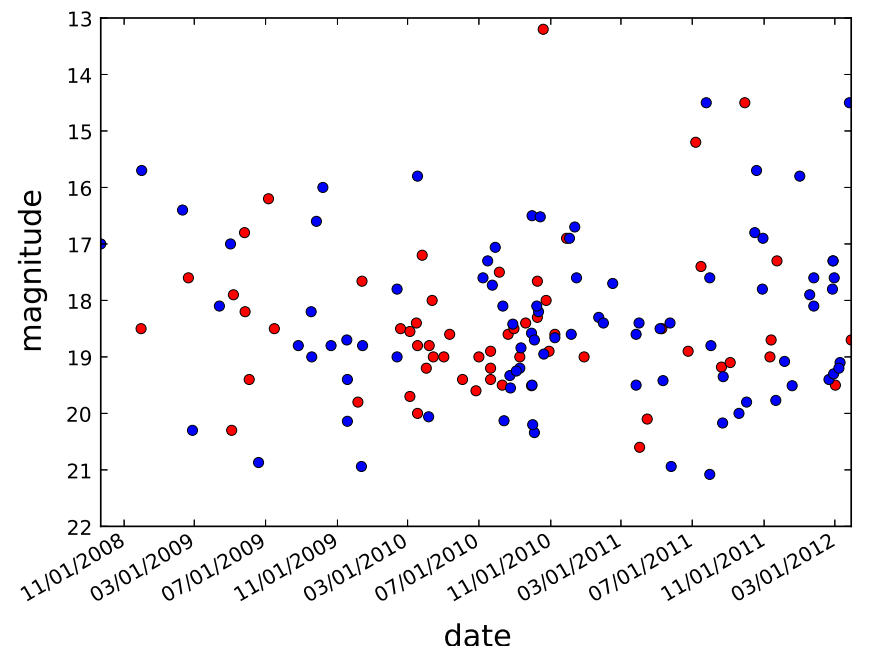

Figure 1. Apparent $R$-band peak (detection) magnitude as a function of the peak (detection) time shown in red (blue) for all $147 \mathrm{SNe}$ in our sample. For some $\mathrm{SNe}$ the peak time and magnitude is not determined; in those cases we use the detection time and magnitude.

with an estimated explosion time later than 2008 August 4 and before 2012 May 1 (this is one year before the end of the studied $\gamma$-ray data sample). The apparent $R$-band peak magnitude $(m)$ as a function of the peak time is shown in Figure 1. Note that throughout this paper we refer to $m$ as the peak magnitude; for sources where the peak magnitude is not determined we use the discovery magnitude instead. The subsample of bright $(m<16.5)$ and/or nearby (with a redshift $z<0.015) \mathrm{SNe}$ used for the joint likelihood analysis is detailed in Table 1.

\section{FERMI LAT $\gamma$-RAY DATA ANALYSIS}

The Fermi LAT is a pair-conversion telescope, sensitive to $\gamma$-rays with energies from $20 \mathrm{MeV}$ to greater than $300 \mathrm{GeV}$ (Atwood et al. 2009). It has a large field of view and has been scanning the entire sky every few hours for the last 6 years. Thus it is very well suited for searches for transient $\gamma$-ray signals on the timescale of months. Bright SN events may be detectable at distances $d<30 \mathrm{Mpc}$ (Murase et al. 2011) depending on the properties of the source. Margutti et al. (2014) searched for $\gamma$-rays from a single $\mathrm{SN}$ in the case of SN2009ip, located at a distance of $24 \mathrm{Mpc}$. No $\gamma$-ray excess was identified in Fermi LAT data at the SN position; this is consistent with the picture of ejecta colliding with a compact and dense but low-mass shell of material. For a detection of a single source, closer and/or brighter $\mathrm{SNe}$ are needed (i.e., reached by larger dissipation and larger CSM masses). The properties of the ejecta and CSM can be estimated from multiwavelength observations in a few cases (e.g., SN2009ip, Ofek et al. 2013), but are uncertain or not known in most cases.

In this analysis we use 57 months of Fermi LAT data recorded between 2008 August 4 and 2013 May 1 (Fermi Mission Elapsed Time 239557418-389092331 s), restricted to the Pass 7 Reprocessed Source class. ${ }^{62}$ We select the standard good time intervals (e.g., excluding time intervals when the field of view of the LAT intersected the earth). The Pass 7 Reprocessed data benefit from an updated calibration

\footnotetext{
62 http://fermi.gsfc.nasa.gov/ssc/data/analysis/documentation/ Pass7REP_usage.html
}

that improves the energy measurement and event-direction reconstruction accuracy at energies above $1 \mathrm{GeV}$ (Bregeon et al. 2013). To minimize the contamination from the $\gamma$-rays produced in the upper atmosphere, we select events with zenith angles $<100^{\circ}$. We perform a binned analysis (i.e., binned in space and energy) using the standard Fermi LAT ScienceTools package, version v09r32p05, available from the Fermi Science Support Center $^{63}$ (FSSC) using the P7REP SOURCE_V15 instrument response functions. We analyze data in the energy range of $100 \mathrm{MeV}$ to $300 \mathrm{GeV}$, binned into 20 logarithmic energy intervals. For each source we select a $20^{\circ} \times 20^{\circ}$ region of interest (ROI) centered on the source localization binned in 0.2 size pixels. The binning is applied in celestial coordinates and an Aitoff projection was used.

We use four different approaches in our analysis.

1. We perform a likelihood analysis to search for $\gamma$-ray excesses that are consistent with originating from a point source coincident with the position of each SNe IIn in our sample over a one-year time scale. We assume that their $\gamma$-ray emission follows a power-law spectrum. This approach is sensitive to single bright sources.

2. In a model-independent approach (i.e., no prior assumption on the SN $\gamma$-ray spectral shape) we compute the likelihood in bins of energy (bin-by-bin likelihood). We use the bin-by-bin likelihood to evaluate $95 \%$ confidence level (CL) flux upper limits in 20 energy bins for the 16 closest and optically brightest $\mathrm{SNe}$ in our sample.

3. In order to increase the sensitivity for a weak signal, we combine individual sources in a joint likelihood analysis using the composite likelihood tool, Composite2, of the Fermi Science Tools.

4. We repeat the joint likelihood analysis using the composite likelihood tool, but limit the sample to those SNe IIn that exhibit additional indications of strong interactions with their CSM. Not all SNe IIn might be surrounded by a massive CSM. This clean sample of SNe with a confirmed massive CSM might produce a strong $\gamma$-ray signal and should provide an enhanced signal-to-background ratio.

Accurate $\mathrm{SN}$ positions are given by optical localizations. Theoretical predictions of the duration of the $\gamma$-ray emission are uncertain and motivate a search in several time windows. We test three different time windows: $\Delta T=1$ year, 6 months, and 3 months. The optical light curve is produced by the interaction of the SN ejecta with the dense CSM and is thus correlated with the expected $\gamma$-ray emission. Most of the $\gamma$-ray emission is expected during the interactions after the shock breakout. The optical light curve peak is reached around the end of the breakout (see, e.g. Ofek et al. 2010). We collected the SN properties from the PTF sample, Astronomer's Telegrams, ${ }^{64}$ and the Central Bureau for Astronomical Telegrams. ${ }^{65}$ Most PTF sources are unpublished and the other events were drawn from ATEL and CBET. Full details and final analysis of the PTF SN IIn sample will be provided in a forthcoming publication. In some cases the known SN properties include the optical flux peak time while in other cases this information is missing and only the optical detection time is available. To account for the uncertainty in the determination of the peak time and to make sure no early $\gamma$-ray emission is missed, we

\footnotetext{
63 http://fermi.gsfc.nasa.gov/ssc/data/analysis/

$64 \mathrm{http}: / / \mathrm{www}$.astronomerstelegram.org/

65 http://www.cbat.eps.harvard.edu/cbet/RecentCBETs.html
} 
Table 1

List of Nearby and/or Bright SNe—with Redshift $z<0.015$ and/or $R$-band Magnitude $m<16.5$

\begin{tabular}{|c|c|c|c|c|c|c|}
\hline Name & R.A. $\left({ }^{\circ}\right)^{\mathrm{a}}$ & Decl. $\left({ }^{\circ}\right)^{\mathrm{a}}$ & Date & $z$ & $m$ & TS ( $p$-value) \\
\hline SN 2008gm & 348.55 & -2.78 & 2008 Oct $22^{\mathrm{b}}$ & 0.012 & $17.00^{\mathrm{c}}$ & $3.2(0.169)$ \\
\hline SN 2008ip & 194.46 & 36.38 & $2008 \operatorname{Dec} 31^{\mathrm{b}}$ & 0.015 & $15.70^{\mathrm{c}}$ & $0.0(0.572)$ \\
\hline SN 2009au & 194.94 & -29.60 & 2009 Mar $11^{\mathrm{b}}$ & 0.009 & $16.40^{\mathrm{c}}$ & $0.0(0.572)$ \\
\hline PTF 10ujc & 353.63 & 22.35 & 2009 Aug 05 & 0.032 & 16.20 & $0.0(0.572)$ \\
\hline SN $2009 \mathrm{kr}$ & 78.01 & -15.70 & 2009 Nov $06^{\mathrm{b}}$ & 0.006 & $16.00^{\mathrm{c}}$ & $4.7(0.104)$ \\
\hline SN 2010bt & 192.08 & -34.95 & 2010 Apr $17^{\mathrm{b}}$ & 0.016 & $15.80^{\mathrm{c}}$ & $14.4(0.0065)$ \\
\hline PTF 10aaxf & 145.72 & 9.50 & 2010 Nov 18 & 0.011 & 13.20 & $7.1(0.039)$ \\
\hline \multicolumn{7}{|l|}{ SN 2010j1 } \\
\hline PTF 10aaxi & 94.13 & -21.41 & 2010 Nov 23 & 0.010 & 18.00 & $0.0(0.572)$ \\
\hline \multicolumn{7}{|l|}{ SN 2010jp } \\
\hline SN 2011A & 195.25 & -14.53 & 2011 Jan $02^{\mathrm{b}}$ & 0.009 & $16.90^{\mathrm{c}}$ & $0.0(0.572)$ \\
\hline PTF 11iqb & 8.52 & -9.70 & 2011 Aug 06 & 0.013 & 15.20 & $0.3(0.469)$ \\
\hline SN 2011fh & 194.06 & -29.50 & 2011 Aug $24^{\mathrm{b}}$ & 0.008 & $14.50^{\mathrm{c}}$ & $1.9(0.262)$ \\
\hline PSNJ $10081059+5150570$ & 152.04 & 51.85 & 2011 Oct 29 & 0.004 & 14.50 & $0.0(0.572)$ \\
\hline \multicolumn{7}{|l|}{ SN 2011ht } \\
\hline PTF 11qnf & 86.23 & 69.15 & 2011 Nov $01^{\mathrm{b}}$ & 0.014 & $19.80^{\mathrm{c}}$ & $1.4(0.320)$ \\
\hline SN 2011hw & 336.56 & 34.22 & 2011 Nov $18^{\mathrm{b}}$ & 0.023 & $15.70^{\mathrm{c}}$ & $0.0(0.572)$ \\
\hline SN 2012ab & 185.70 & 5.61 & 2012 Jan $31^{\mathrm{b}}$ & 0.018 & $15.80^{\mathrm{c}}$ & $0.0(0.572)$ \\
\hline PSNJ 18410706-4147374 & 280.28 & -41.79 & 2012 Apr $25^{\mathrm{b}}$ & 0.019 & $14.50^{\mathrm{c}}$ & $0.0(0.572)$ \\
\hline SN 2012ca & & & & & & \\
\hline
\end{tabular}

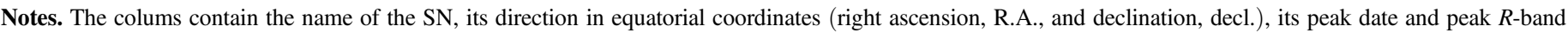

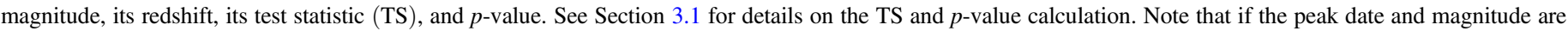
not available in the catalog, the discovery date and magnitude are quoted instead.

a Epoch J2000.0.

${ }^{\mathrm{b}}$ Discovery date.

${ }^{\mathrm{c}}$ Discovery magnitude.

start the time window 30 days before the peak time (or the detection time in case the peak time is not provided). In the case of the three novae, the reported $\gamma$-ray light curves (see Figure 1 in Hill et al. 2013) have very similar durations, justifying a similar time window for all sources. However, the duration of the novae detected by Fermi were $\sim 20$ days, while SN IIn typically last longer, $\mathcal{O}(100$ days -1 year $)$.

\subsection{Source Specific Analysis}

We analyze the $20^{\circ} \times 20^{\circ}$ ROI around each source in our SN sample in a one-year time window in a binned likelihood analysis. We construct a model whose free parameters are fitted to the data in the ROI. This model includes a point-like source at the $\mathrm{SN}$ position; its $\gamma$-ray spectrum is represented as a powerlaw function with both index and normalization free to vary. In addition we have to model the point sources in the ROI and the diffuse $\gamma$-ray emission. We consider all the 2FGL sources (Nolan et al. 2012) included within a larger region of radius, $R=20^{\circ}$, to allow for the breadth of the LAT point-spread function that may cause a significant signal from sources outside the ROI to leak into it. The positions and spectral parameters of all $2 \mathrm{FGL}$ sources within $15^{\circ}<R<20^{\circ}$ from the center of the ROI are fixed to the values reported in the 2FGL catalog; those are on average 21 sources. For the sources within $5^{\circ}<R<15^{\circ}$ with $>15 \sigma$ detection significance in 2FGL only the flux normalization is left free to vary and all the other parameters are fixed to the values reported in the 2FGL catalog. The parameters for all the other sources within $5^{\circ}<R<15^{\circ}$ are fixed to the 2FGL catalog values. Finally, for sources within $R<5^{\circ}$ all parameters (index and normalization in case of a power-law spectrum; index, cutoff, and normalization in case of a power-law with exponential cutoff and normalization; spectral slope and curvature in case of a log-parabola source spectrum) are free to vary if the source significance exceeds $4 \sigma$, otherwise all source parameters are fixed. On average 3 sources per ROI have all parameters free, while 6 sources have a free normalization and 18 sources are fixed to the 2FGL values.

We determine the best values for all the free parameters, fitting our source model together with a template for the isotropic and Galactic interstellar emission ${ }^{66}$ to the LAT data with a binned likelihood approach as described in Abdo et al. (2009). To quantify the significance of a potential excess above the background, we employ the likelihood-ratio test (Neyman \& Pearson 1928). We form a test statistic

$$
\mathrm{TS}=-2 \Delta \log \mathcal{L}=-2\left(\log \mathcal{L}_{0}-\log \mathcal{L}\right),
$$

where $\mathcal{L}_{0}$ is the likelihood evaluated at the best-fit parameters under a background-only, null hypothesis, i.e., a model that does not include a point source at the $\mathrm{SN}$ position, and $\mathcal{L}$ is the likelihood evaluated at the best-fit model parameters when including a candidate point source at the SN position.

The distribution of the TS values obtained for all the SNs using a one-year time window is displayed in Figure 2 (left), compared to the TS distribution obtained from performing a similar analysis at random positions in the sky. We require the random ROI centers to be separated by at least $3: 5$ and to lie outside of the Galactic plane, i.e., $|b|>10^{\circ}$. The analysis in the Galactic plane region is complicated by the intense Galactic

\footnotetext{
${ }^{66} \mathrm{We}$ use the templates provided by the FSSC for the P7REP SOURCE_V15 event class (http://fermi.gsfc.nasa.gov/ssc/data/access/lat/ BackgroundModels.html) with free normalization and free index in case of the Galactic interstellar emission model.
} 

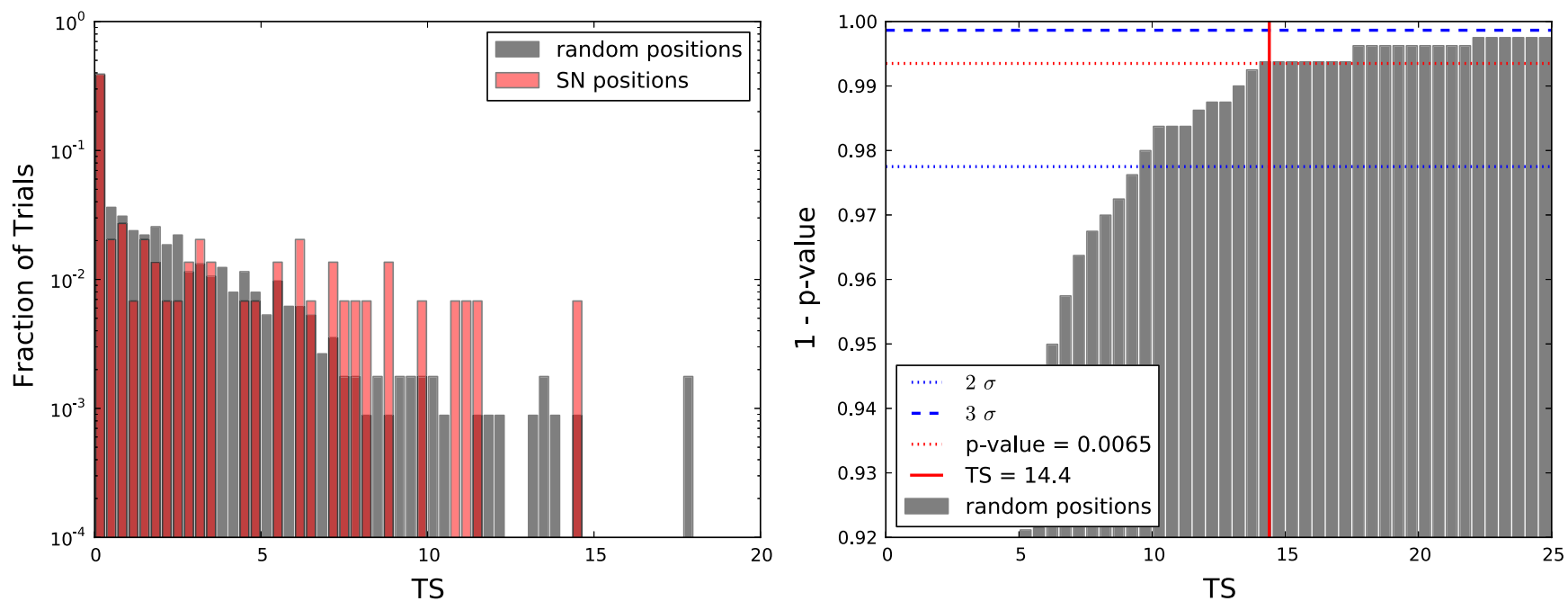

Figure 2. Left: distribution of TS values for a test source modeled by a power-law energy spectrum located at an SN position (red), compared to TS for a similar test source located at a random position (gray). Right: cumulative distribution of random-position TS values. The blue dashed (dotted) line indicates a Gaussian equivalent one-sided $3 \sigma(2 \sigma)$ probability of finding a larger TS than the TS indicated by the intersection of the blue line with the gray distribution. The red solid line shows the largest TS found in the source-specific analysis, which has a $p$-value of 0.0065 (red dotted line) and thus lies below $3 \sigma$. Considering the trials factor, the $p$-value increases to 0.6 .
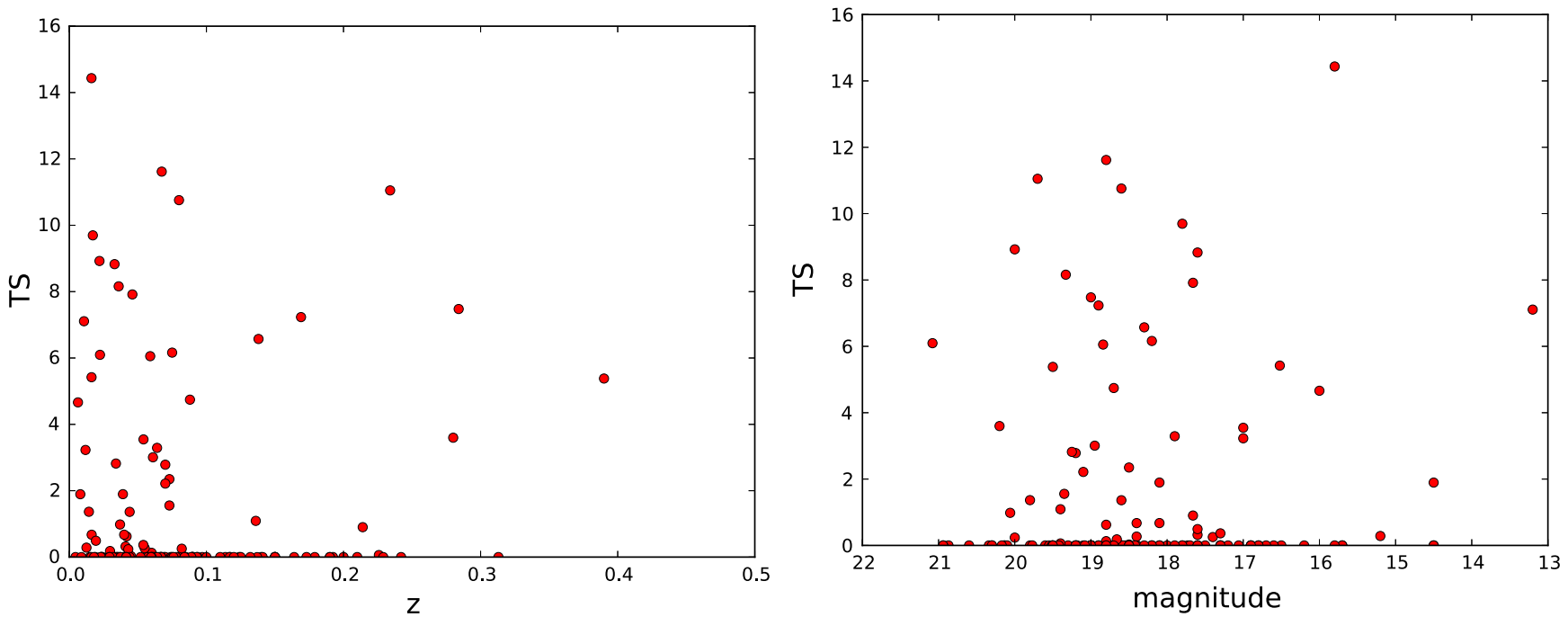

Figure 3. TS of a test source located at the SN position as a function of redshift (left) and as a function of magnitude (right). Note that the largest TS value was 14.4 (corresponding to a $p$-value of 0.009 ), which does not exceed the detection threshold of $5 \sigma$.

diffuse emission and none of the $\mathrm{SNe}$ in our sample are located close to the plane. Those requirements limit the number of independent ROIs; we use 1140 ROIs in our analysis. The distribution of SN-position TS values is similar to the distribution of random-position TS values (see Figure 2 left). The highest TS value found among the SN positions is 14.4, which corresponds to a $p$-value of 0.0065 (obtained from the random position analysis), which is below $3 \sigma$ for a single trial (see Figure 2 right). Given the number of SNe in our sample a trials factor needs to be applied, which increases the $p$-value to 0.6.

Optically bright $\mathrm{SNe}$ are expected to produce a brighter $\gamma$-ray signal than optically dim ones and nearby $\mathrm{SNe}$ are expected to be brighter than sources at large distance. However, we do not find an obvious correlation of TS value with redshift or magnitude (see the left and right panels of Figure 3, respectively), indicating that the $\gamma$-ray signals of individual SNe, if present, are weak.

Three of the $147 \mathrm{SNe}$ have a 2FGL source in their close vicinity with an angular distance of less than $0: 4$. In each case the nearest 2FGL source is associated with an active galactic nucleus through multi-wavelength data. Since the spectral parameters of the nearby source are left free to vary in the fit, a possible SNe flux could have been absorbed by the background source. Those sources are PTF 10weh, LSQ 12by and SN 2012bq, which are optically dim and distant sources and thus not part of the subsample of nearby and/or bright SNe.

\subsection{Model-independent Analysis of Nearby and/or Bright SNe}

The $\gamma$-ray spectral shape resulting from particle acceleration in the interaction of SN ejecta with a dense CSM is not known a priori. It is determined by the initial proton spectrum and could be altered by the absorption of the $\gamma$-rays in the surrounding medium. Therefore, we study the closest and/or optically brightest sources, which are the most promising sources in terms of expected $\gamma$-ray emission, in an approach independent of an SN spectral model assumption. The sources chosen for this analysis have to fulfill the criteria of $z<0.015$ or $m<16.5$, and are listed in Table 1 . We fix the spectral 


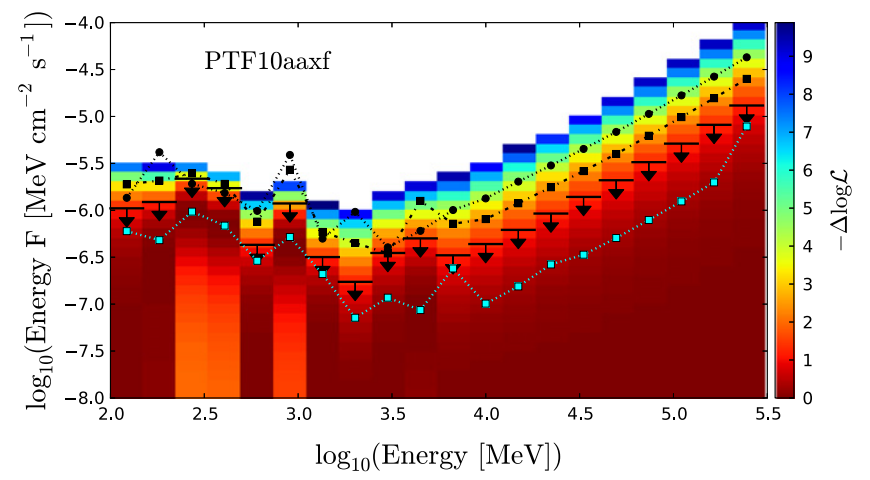

Figure 4. Histogram of the bin-by-bin LAT likelihood function used to test for a putative $\gamma$-ray source at the position of supernova SN 2010j1 (PTF 10aaxf). The bin-by-bin likelihood is calculated by scanning the integrated energy flux of the SN within each energy bin (equivalent to scanning in the spectral normalization of the source). When performing this scan, the flux normalizations of the background sources are fixed to their optimal values as derived from a maximum likelihood fit over the full energy range. Within each bin, the color scale denotes the variation of the logarithm of the likelihood with respect to the best-fit value of the SN flux using a 1 year time window. Upper limits on the integrated energy flux are set at $95 \% \mathrm{CL}$ within each bin using the deltalog-likelihood technique and are largely independent of the SN spectrum. The black arrows indicate the $95 \% \mathrm{CL}$ flux upper limits for $\Delta T=1$ year, where the shown log-likelihood decreases by $2.71 / 2$ from its maximum. For completeness we overlay the $95 \% \mathrm{CL}$ upper limits for $\Delta T=6$ months and $\Delta T=3$ represented by dotted-dashed and dotted lines respectively. For the particular case of SN 2010jl we repeated the analysis for an extended time window spanning 4.5 years. The results are overlaid as cyan dotted line.

parameters of the background sources and the diffuse templates to their global values obtained from the source-by-source analysis over the entire energy range described in Section 3.1 (without including the $\mathrm{SN}$ itself). Following the procedure described in Ackermann et al. (2014) we calculate the likelihood in each of the 20 energy bins after inserting a test source at the SN position at various flux normalization values:

$$
\mathcal{L}\left(\left\{\boldsymbol{\mu}_{j}\right\}, \hat{\boldsymbol{\theta}} \mid \mathcal{D}\right)=\prod_{j} \mathcal{L}_{j}\left(\boldsymbol{\mu}_{j}, \hat{\boldsymbol{\theta}} \mid \mathcal{D}_{j}\right),
$$

where $\mathcal{D}_{j}$ is the photon data, $\mathcal{L}_{j}$ is the Poisson likelihood, and $\left\{\boldsymbol{\mu}_{j}\right\}$ is a set of independent signal parameters in energy bin $j$. The symbol $\boldsymbol{\theta}$ represents the nuisance parameter (i.e., free parameters of background sources and diffuse templates) and $\hat{\boldsymbol{\theta}}$ indicates that they have been fixed to their global values. The binby-bin likelihood allows us to find the upper limits at 95\% CL, ${ }^{67}$ defined as the value of the energy flux, where the log-likelihood decreases by $2.71 / 2$ from its maximum (the "delta-log-likelihood technique"-Bartlett 1953; Rolke et al. 2005). An example is shown in Figure 4 for SN 2010jl (PTF 10aaxf-Zhang et al. 2012; Fransson et al. 2014; Ofek et al. 2014b), while similar plots for all nearby sources can be found in Appendix B. Any SN model predicting a certain $\gamma$-ray spectrum can be tested using those results (see Ackermann et al. 2014, for more details on the bin-by-bin likelihood) by recreating a global likelihood by tying together the signal parameters over the energy bins:

$$
\mathcal{L}(\boldsymbol{\mu}, \hat{\boldsymbol{\theta}} \mid \mathcal{D})=\prod_{j} \mathcal{L}_{j}\left(\boldsymbol{\mu}_{j}(\boldsymbol{\mu}), \hat{\boldsymbol{\theta}} \mid \mathcal{D}_{j}\right),
$$

with $\boldsymbol{\mu}$ denoting the global signal parameters.

\footnotetext{
67 Note, we are using a two-sided confidence interval.
}

For the most promising source of our sample, SN 2010j1, we repeat the analysis for an extended time window ending in 2015 May, i.e., spanning 4.5 years. This is motivated by the fact that in some cases SN Type IIn emission lasts for 3-5 years after the explosion (Cooke et al. 2009).

\subsection{Joint Likelihood Analysis}

For greater sensitivity to a weak $\gamma$-ray signal from interaction-powered $\mathrm{SNe}$, we combine the 16 closest and/or brightest sources in a joint likelihood analysis. To be independent from any spectral shape assumption we perform the analysis in energy bins (see Section 3.2 for details of the bin-by-bin likelihood analysis). In each energy bin we tie the $\mathrm{SN}$ flux normalization for all $16 \mathrm{SNe}$ together resulting in one free parameter per energy bin. The likelihood values for the individual sources, $i$, are multiplied to form the joint likelihood

$$
\mathcal{L}\left(\boldsymbol{\mu},\left\{\hat{\boldsymbol{\theta}}_{i}\right\} \mid \mathcal{D}\right)=\prod_{i} \mathcal{L}_{i}\left(\boldsymbol{\mu}, \hat{\boldsymbol{\theta}}_{i} \mid \mathcal{D}_{i}\right) .
$$

However, we have to make some assumption about a common scaling factor of the $\gamma$-ray flux in order to tie the SNe flux normalizations together (i.e., we want to give a larger weight to SNe with greater expected $\gamma$-ray fluxes in the joint likelihood). We use two different approaches: first, we assume that all SNe have the same intrinsic $\gamma$-ray luminosity; therefore, the observed $\gamma$-ray flux for each SN scales with a factor inversely proportional to the square of the luminosity-distance $d$. The redshift is measured for each $\mathrm{SNe}$ and since we only consider nearby $\mathrm{SNe}$ we use a simple linear approximation for the relation between redshift and distance: $d=z \times c / H$, with $H=67.8 \mathrm{~km} \mathrm{~s}^{-1} \mathrm{Mpc}^{-1}$ (Ade et al. 2014). We do not apply a redshift-dependent energy rescaling for $\mathrm{SNe}$ at different redshifts, since the energy shift is negligible at the small redshifts (i.e., $z<0.015$ ) considered in this analysis. We weight the flux normalization in each energy bin of each source with $w_{d}=(10 \mathrm{Mpc} / d)^{2}$. We then tie those weighted normalizations together. The exact value of $H$ does not influence our results since the combined normalization of all sources is free in the fit of the model to the data in each energy bin. Note that only the SN flux normalization is free while the background source parameters as well as the diffuse template parameters are fixed to their global values obtained from a fit to the entire energy range.

Alternatively, we assume that the $\gamma$-ray flux is correlated with the optical flux, i.e., we use a weight proportional to the optical flux ${ }^{68}$ or $10^{-0.4 m}$. We chose the weight to be

$$
w_{m}=10^{-0.4(m-C)}=10^{-0.4 m+5.2},
$$

where $m$ is the apparent $R$-band magnitude provided by the SN catalog and $C=13$ is a normalization constant. Again, the exact choice of $C$ does not influence our results since the combined normalization of all sources is free in the fit. We chose to neglect a correction for Galactic dust extinction, which is at most $0.28 \mathrm{mag}$ and thus smaller than the uncertainty in the peak magnitude determination.

\footnotetext{
${ }^{68}$ Note that flux and apparent magnitude are related through $m-m_{0}=-2.5 \log _{10} \frac{F}{F_{0}}$, where $F_{0}$ and $m_{0}$ are the flux and apparent magnitude of a reference star.
} 

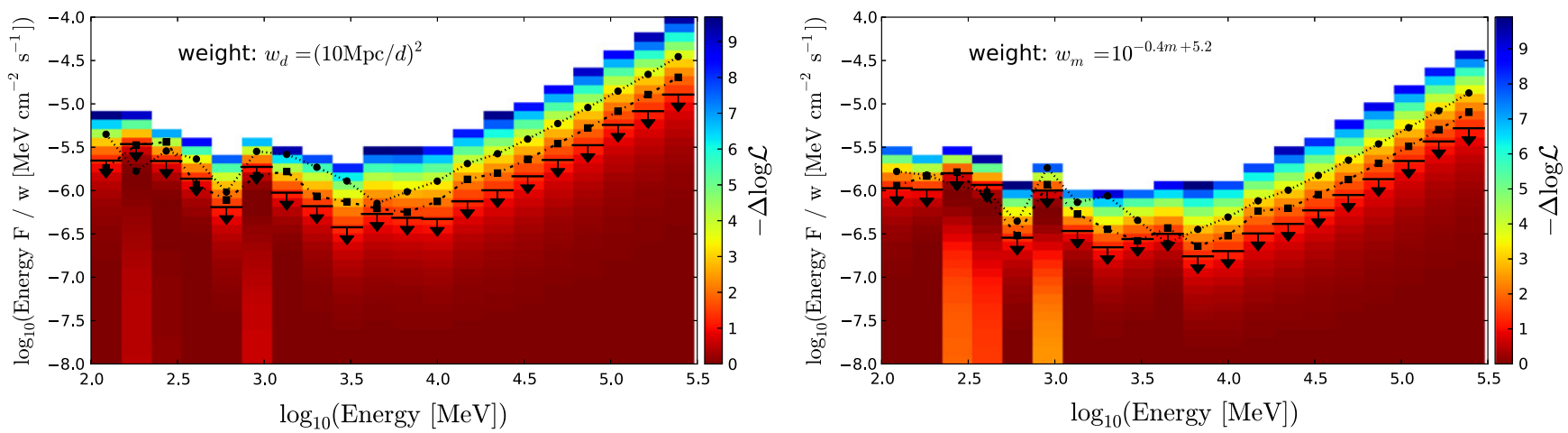

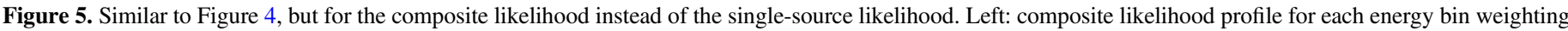

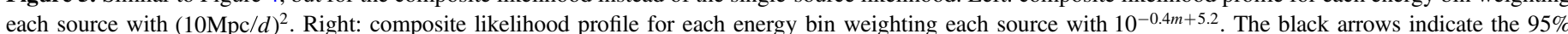
upper limits for $\Delta T=1$ year, while the dotted-dashed and dotted lines represent the $95 \%$ upper limits for $\Delta T=6$ months and $\Delta T=3$ months, respectively.

Table 2

Sum over Bin-by-bin TS Values Obtained from the Joint Likelihood Analysis

\begin{tabular}{|c|c|c|c|c|c|c|}
\hline \multirow[t]{2}{*}{ Weighting } & \multicolumn{3}{|c|}{$\mathrm{TS}$} & \multicolumn{3}{|c|}{$\mathrm{TS}_{\mathrm{PL}}(p$-value $)$} \\
\hline & 1 year & 6 months & 3 months & 1 year & 6 months & 3 months \\
\hline$\overline{(10 \mathrm{Mpc} / d)^{2}}$ & 2.2 & 2.1 & 2.4 & $0.0(1.0)$ & $0.0(1.0)$ & $0.0(1.0)$ \\
\hline $10^{-0.4 m+5.2}$ & 11.7 & 7.8 & 9.0 & $2.9(0.23)$ & $1.6(0.45)$ & $0.0(1.0)$ \\
\hline
\end{tabular}

Note. $\mathrm{TS}_{\mathrm{PL}}$ is the TS obtained by assuming a power-law spectral shape.

We perform the joint likelihood analysis for three time windows: 1 year, 6 months, and 3 months since the $R$-band maximum light. Figure 5 shows the likelihood profiles of the combined $\gamma$-ray flux. Table 2 summarizes the results from the combined likelihood analysis and shows the sum of TS over all energy bins. No significant improvement in the likelihood by including the $\mathrm{SNe}$ in the fit could be found in the joint likelihood analysis. The largest TS value of 8.8 is found in case of assuming the $\gamma$-ray flux scales with the optical flux for the one-year time windows. According to Wilks' theorem, TS is distributed approximately as $\chi^{2}$ with the degrees of freedom equal to the number of parameters characterizing the additional source. Taking into account the number of free parameters $(20$, one for each energy bin) the probability that this is a statistical fluctuation is $98.5 \%$. This significance would be further decreased by taking into account trial factors for the two different weighting schemes and 3 different time windows.

However, if we assume a spectral model for the SN flux, we can greatly reduce the number of free parameters. For illustration we fit a power-law spectral shape to the bin-bybin likelihood following Equation (3). The index and normalization of the power-law function are left free to vary in the fit. The resulting TS values and corresponding $p$-values (not including trials factors) are summarized in Table 2; none of them are significant. A more physical spectral model is fitted to the bin-by-bin likelihood in Section 4.

\subsection{Joint Likelihood Analysis of SN Subsample with Confirmed Massive CSM}

We select a subsample of $16 \mathrm{SNe}$ from the $\mathrm{SNe}$ IIn catalog for which we have additional evidence through multiwavelength observations for the existence of a massive CSM. We select SNe that show Balmer emission lines and continuum in both early and late times. The SNe in this sample are: PTF 12csy, PTF 11oxu, PTF 11mhr, PTF 11fzz, PTF 11 fuu, PTF 10aaxf, PTF 10ptz, PTF 10scc, PTF 10jop, PTF 10fei, PTF

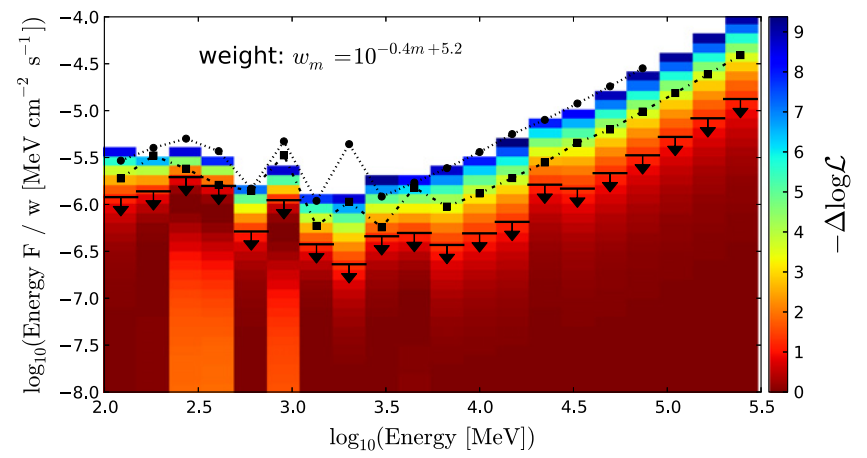

Figure 6. Joint likelihood analysis of the $\mathrm{SN}$ subsample with a confirmed massive CSM: joint likelihood profile for each energy bin weighting each source with $10^{-0.4 m+5.2}$. The black arrows indicate the $95 \%$ upper limits for $\Delta T=1$ year, while the dotted-dashed and dotted lines represent the $95 \%$ upper limits for $\Delta T=6$ months and $\Delta T=3$ months, respectively.

10qaf, PTF 10tel, PTF 10tyd, PTF 10gvf, PTF 10cwl, PTF $09 \mathrm{drs}$. We repeat the joint likelihood analysis described above for this subset with the optical flux weighting scheme for three time windows ( 1 year, 6 months, and 3 months). The results are displayed in Figure 6. The TS values of the composite fit are $11.3,17.5$, and 10.3 for the time windows of 1 year, 6 months, and 3 months, respectively. Taking into account the 20 free parameters, the chance probability for a TS of 17.5 is $62 \%$.

\section{INTERPRETATION}

Murase et al. (2011) suggested that $\gamma$-ray emission is produced by cosmic rays accelerated at the early collisionless shock between SN ejecta and circumstellar material. For the scenario described by Murase et al. (2014), $\gamma$-ray emission can be predicted when the model parameters are determined by optical and X-ray observations. We defer such modeldependent analyses to future work. Instead, in this work, we take a model-independent approach, where we aim to constrain 

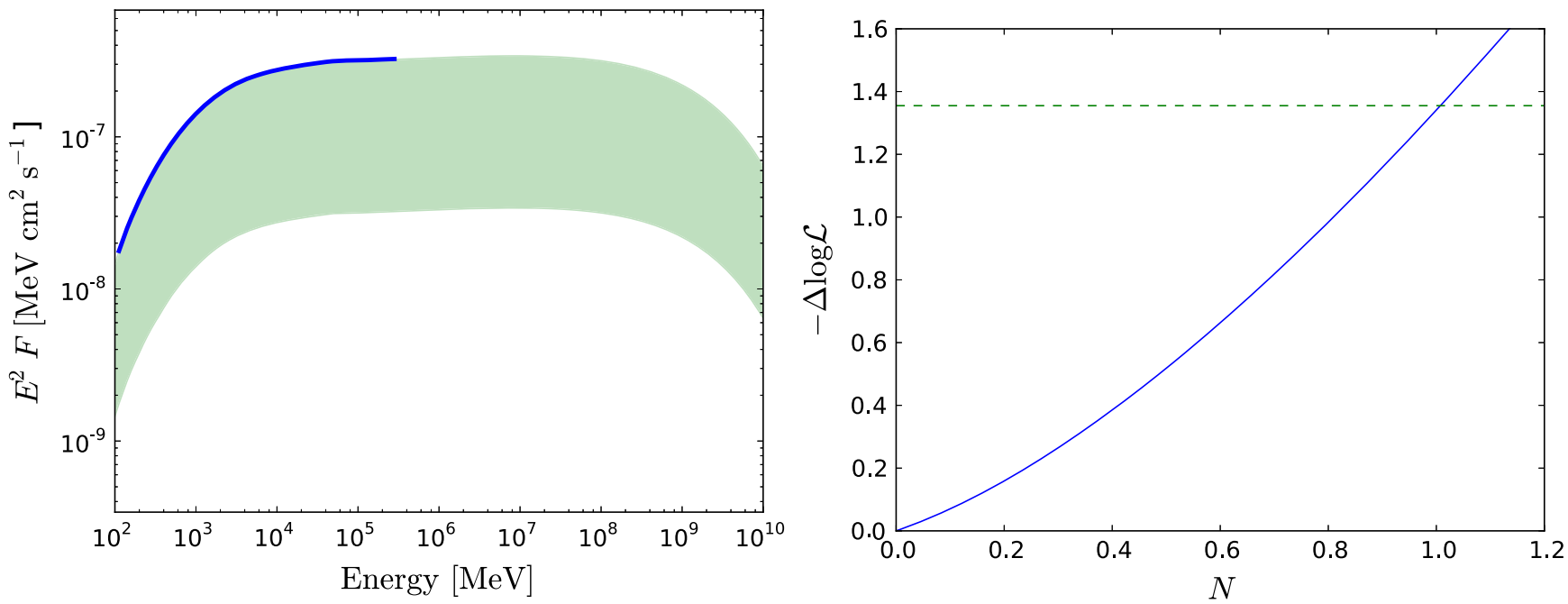

Figure 7. Left: predicted $\gamma$-ray energy spectrum for SN 2010jl assuming $\Gamma_{\mathrm{p}}=-2$ and a normalization of the $\gamma$-ray flux yielding $0.01<L_{\gamma} / L_{R}<0.1$ shown as the green shaded region compared to the $95 \%$ flux upper limit (blue). Right: likelihood profile for the spectral normalization parameter $N$ relative to the flux prediction yielding $L_{\gamma} / L_{R}=0.1$. The dashed green line indicates an increase of the negative delta log-likelihood by $2.71 / 2$ compared to its minimum.

the $\gamma$-ray luminosity as a function of the proton spectral index. We assume that the spectrum of $\mathrm{CR}$ protons is given by a power law (in momentum) with minimum and maximum proton momenta of 0.1 and $10^{8} \mathrm{GeV} \mathrm{c}^{-1}$, respectively. Then, we calculate the $\gamma$-ray flux following Kelner et al. (2006). In the calorimetric limit, which is expected for SNe like SN 2010j1 (Murase et al. 2014), the $\gamma$-ray spectral index follows the proton spectral index, although the resulting limits (shown in Figure 9) are similar to what would be obtained for noncalorimetric cases, for which the resulting shape of the $\gamma$-ray spectrum is slightly harder than the proton spectral shape due to the energy dependence of the pp cross section. For simplicity we do not take into account $\gamma$-ray absorption; Murase et al. (2014) showed that $\mathrm{GeV} \gamma$-rays can escape from the system without severe matter attenuation if the shock velocity is high enough.

The diffusive shock acceleration theory predicts that the proton acceleration efficiency is $\epsilon_{\mathrm{p}} \sim 0.1$. In the calorimetric limit, all the proton energy is used for pion production, and $1 / 3$ of pions are neutral pions that decay into $\gamma$-rays. Then about half of the $\gamma$-rays are absorbed deep inside the ejecta, so we expect $L_{\gamma} \approx(1 / 6) \epsilon_{\mathrm{p}} f_{\text {esc }} L_{\text {kin }}$, where $L_{\text {kin }}$ is the kinetic luminosity and $f_{\text {esc }}$ is the escape fraction of $\gamma$-rays. The $\gamma$-ray attenuation due to the Bethe-Heitler process is relevant when the shock velocity is lower than $\sim 4500 \mathrm{~km} \mathrm{~s}^{-1}$, while the twophoton annihilation process is relevant when the shock velocity is high enough (Murase et al. 2014). Although $\gamma$-rays can escape late after the shock breakout, the attenuation can be relevant around the shock breakout so we assume $f_{\text {esc }} \sim 0.1-1$ to take into account uncertainty of the $\gamma$-ray flux. The radiation energy fraction is given by $\epsilon_{\gamma} \equiv L_{\mathrm{rad}} / L_{\mathrm{kin}}$, where $L_{\mathrm{rad}}$ is the bolometric radiation luminosity. About half of the kinetic energy is converted into the thermal energy, and half of the thermal energy is released as outgoing radiation, which implies $\epsilon_{\gamma} \sim 1 / 4$ (Ofek et al. 2014b). As a result, we have $L_{\gamma} / L_{\mathrm{rad}} \approx(1 / 6)\left(\epsilon_{\mathrm{p}} / \epsilon_{\gamma}\right) f_{\mathrm{esc}} \sim(1 / 15) f_{\text {esc }}$. Our limits presented below are on the fraction of $\gamma$-ray to $R$-band luminosity, which is an upper bound on $L_{\gamma} / L_{\mathrm{rad}}$. In the case of SN 2010j1 $L_{R} \sim L_{\mathrm{rad}}$ and thus $L_{\gamma} / L_{R} \sim 0.01-0.1$ is theoretically expected.
As an example, we consider supernova SN 2010j1 (PTF 10aaxf), which is the most-likely detectable CR accelerator, because multi-wavelength observations indicate a very massive CSM of $10 M_{\odot}$. We present a generic flux prediction for the calorimetric limit for this source assuming a proton spectral index of $\Gamma_{\mathrm{p}}=-2$ and a normalization of the $\gamma$-ray flux that yields $0.01<L_{\gamma} / L_{R}<0.1$ (shown as shaded green region in Figure 7) and calculate the corresponding flux upper limit (shown in blue in Figure 7) following the procedure outlined in Ackermann et al. (2014). The bin-by-bin likelihood analysis is used to re-create a global likelihood for a given signal spectrum by tying the signal parameters across the energy bins (see Equation (3)). In this case the global signal parameter is the flux scale factor $N$ relative to the flux that yields $L_{\gamma} / L_{R}=0.1$ (i.e., the upper bound of the uncertainty band shown in Figure 7, left). We assume that SN 2010jl is at distance 48.7 Mpc with an apparent $R$-band peak magnitude of 13.2. We calculate the change in log-likelihood for various values of $N$ and find the 95\% flux upper limit (given by the value of $N$ for which the delta log-likelihood decreases by $2.71 / 2$ compared to its minimum). The derived upper limit touches the optimistic model prediction, i.e., the upper bound of the theoretical uncertainty band. A more detailed modeling of the expected flux based on multi-wavelength observations is outside the scope of this paper and will follow in future work. Better constraints on the $\gamma$-ray escape fraction are crucial to calculate stringent limits on the proton acceleration efficiency and will be obtained in more detailed modeling.

More stringent limits are expected from the joint likelihood results. ${ }^{69}$ Generic $\gamma$-ray flux predictions for various proton spectral indices are shown in Figure 8. We calculate the 95\% CL upper limit on the $\gamma$-ray luminosity

$$
L_{\gamma}=4 \pi d^{2} F_{\gamma}^{I}=4 \pi(10 \mathrm{Mpc})^{2} \frac{F_{\gamma}^{I}}{w_{d}}
$$

\footnotetext{
$\overline{69}$ Note that including sources with a statistical over-fluctuation can worsen the joint limit.
} 


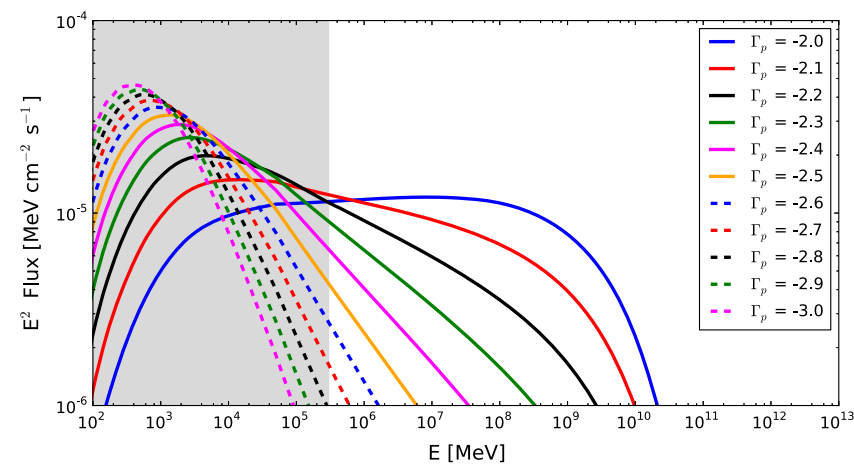

Figure 8. Gamma-ray energy spectra assuming a total $\gamma$-ray energy of $10^{50} \mathrm{erg}$, a source distance of $10 \mathrm{Mpc}$ and a duration of 1 year for various proton spectral indices $\Gamma_{\mathrm{p}}$. The shaded gray region shows the energy range covered by this analysis.

where $F_{\gamma}^{I}$ is the integrated $\gamma$-ray flux over the energy range used in this analysis. The luminosity $L_{\gamma}$ is proportional to the result of the joint likelihood analysis using the weight $w_{d}=(10 \mathrm{Mpc} / d)^{2}$, assuming all sources have the same $L_{\gamma}$. In other words our joint likelihood results set a limit on $F_{\gamma}^{I} / w_{d}$ and thus on $L_{\gamma}$. The result is shown in Figure 9 (left) as a function of the proton spectral index.

In addition we calculate the $95 \%$ CL upper limit on the ratio of $\gamma$-ray to optical luminosity

$$
L_{\gamma} / L_{R}=\frac{4 \pi d^{2} F_{\gamma}^{I}}{L_{\odot} 10^{0.4\left(M_{\odot}-M\right)}}=\frac{4 \pi(1 \mathrm{Mpc})^{2}}{L_{\odot} 10^{0.4 M_{\odot}+4.8}} \frac{F_{\gamma}^{I}}{w_{m}},
$$

where $L_{\odot}=6 \times 10^{32} \mathrm{erg} \mathrm{s}^{-1}$ is the $R$-band luminosity and $M_{\odot}=4.7$ the absolute $R$-band magnitude of the Sun. The ratio is proportional to $F_{\gamma} / w_{m}$, which is constrained by the joint likelihood analysis assuming a correlation of optical and $\gamma$-ray flux, i.e., weighting with $w_{m}=10^{-0.4 m+5.2}$. Thus we can use the joint likelihood results to set a limit on $L_{\gamma} / L_{R}$ as a function of $\Gamma_{\mathrm{p}}$ (see Figure 9 right).

In Figure 9 both limits discussed above are compared to the limit obtained using only one SN. The closest SN (SN 2011ht with a distance of $d=17.7 \mathrm{Mpc}$ ) is discussed in the case of $1 / d^{2}$ weighting and the brightest SN (SN 2010jl with a magnitude of $m=13.2$ ) is discussed in the case of weighting with the optical flux. In both cases the combined limit is dominated by one $\mathrm{SN}$. In the case of $1 / d^{2}$ weighting the single source limit is better than the combined limit, indicating a statistical under-fluctuation in the individual analysis of this source or an over-fluctuation in one of the sources included in the joint likelihood.

\section{CONCLUSIONS}

The origin of the multi-wavelength emission of SNe IIn and the onset of cosmic-ray production in supernova remnants is not fully understood. SNe IIn are expected to be host sites of particle acceleration, which could be pinpointed by transient $\gamma$ ray signals. For the first time we searched in a systematic way for $\gamma$-ray emission from a large ensemble of $\mathrm{SNe}$ In in coincidence with optical signals. No evidence for a signal was found, but our observational limits start to reach interesting parameter ranges expected by the theory. We set stringent limits on the $\gamma$-ray luminosity and the ratio of $\gamma$-ray and optical luminosity. For example, we can exclude $L_{\gamma} / L_{R}>0.1$ at $95 \%$ $\mathrm{CL}$ for proton spectral indices of $<2.7$ from the results of the combined likelihood analysis assuming that $L_{\gamma} / L_{R}$ is constant. Those constraints can be converted to limits on the proton acceleration efficiency. In the case of SN 2010jl, our limits are close to theoretically expected values. However, uncertainties in the modeling, including the $\gamma$-ray escape fraction, lead to the range of $O(10 \%)$ to $O(1 \%)$ for the ratio of $\gamma$-ray to optical luminosity. Model-dependent calculations based on multiwavelength observations will be performed in a future work and will allow us to set stringent constraints on the proton acceleration efficiency.

We do not have to make this assumption in the analysis of individual SNe. The results from the optically brightest SN in our sample, SN 2010jl, alone lead to only a factor of two weaker constraints, excluding $L_{\gamma} / L_{R}>0.2$. Assuming a scaling of the $\gamma$-ray flux with $1 / d^{2}$ we can exclude $L_{\gamma}>4 \times 10^{40} \mathrm{erg} \mathrm{s}^{-1}$ at $95 \% \mathrm{CL}$ for all indices considered. A total $\gamma$-ray luminosity of $10^{50} \mathrm{erg}$ emitted within 1 year (as assumed in Figure 8) is excluded. The limits presented here are based on minimal assumptions about the $\gamma$-ray production and can be used to test various models.

The Fermi-LAT Collaboration acknowledges generous ongoing support from a number of agencies and institutes that have supported both the development and the operation of the LAT as well as scientific data analysis. These include the National Aeronautics and Space Administration and the Department of Energy in the United States, the Commissariat à l'Energie Atomique and the Centre National de la Recherche Scientifique/Institut National de Physique Nucléaire et de Physique des Particules in France, the Agenzia Spaziale Italiana and the Istituto Nazionale di Fisica Nucleare in Italy, the Ministry of Education, Culture, Sports, Science and Technology (MEXT), High Energy Accelerator Research Organization (KEK) and Japan Aerospace Exploration Agency (JAXA) in Japan, and the K.A. Wallenberg Foundation, the Swedish Research Council and the Swedish National Space Board in Sweden. Additional support for science analysis during the operations phase is gratefully acknowledged from the Istituto Nazionale di Astrofisica in Italy and the Centre National d'Études Spatiales in France. This paper is based on observations obtained with the Samuel Oschin Telescope as part of the Palomar Transient Factory project, a scientific collaboration between the California Institute of Technology, Columbia University, Las Cumbres Observatory, the Lawrence Berkeley National Laboratory, the National Energy Research Scientific Computing Center, the University of Oxford, and the Weizmann Institute of Science. Some of the data presented herein were obtained at the W.M. Keck Observatory, which is operated as a scientific partnership among the California Institute of Technology, the University of California, and NASA; the Observatory was made possible by the generous financial support of the W.M. Keck Foundation. We are grateful for excellent staff assistance at the Palomar, Lick, and Keck Observatories. E.O.O. is the incumbent of the Arye Dissentshik career development chair and is grateful for support by grants from the Willner Family Leadership Institute Ilan Gluzman (Secaucus NJ), the Israeli Ministry of Science, the Israel Science Foundation, Minerva and the I-CORE 

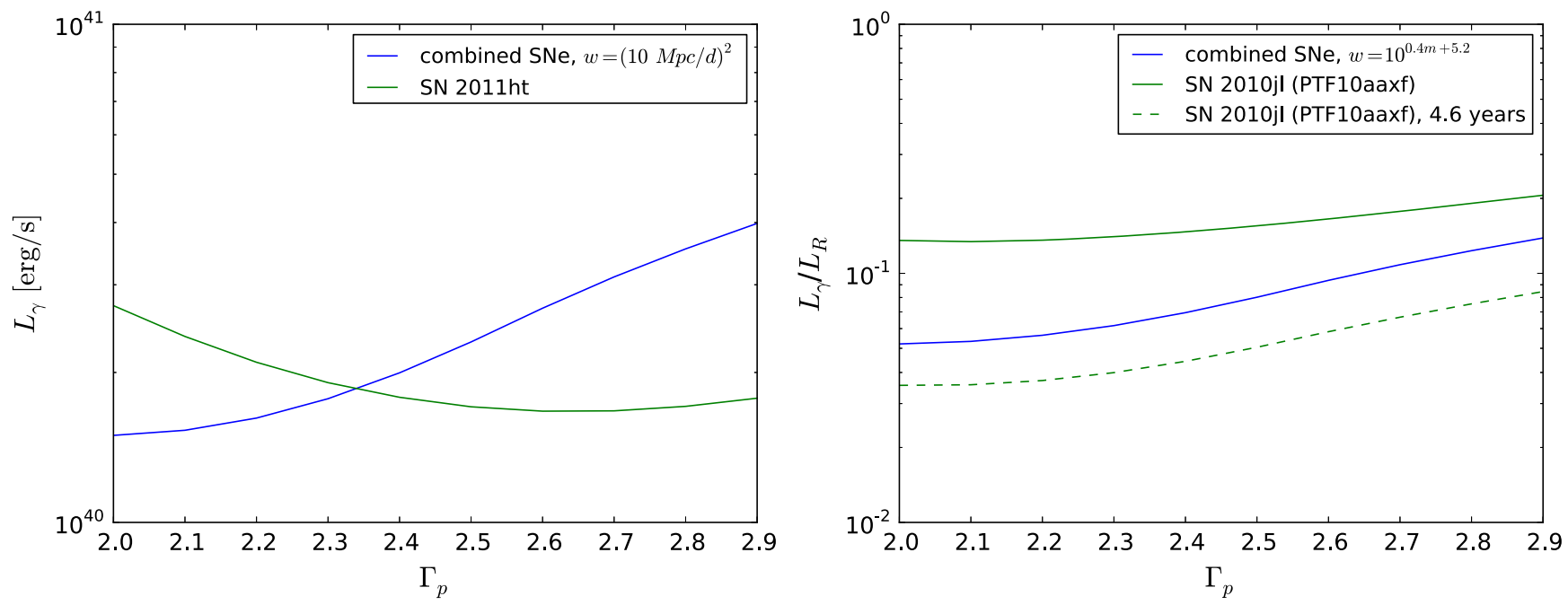

Figure 9. Left: 95\% CL upper limit on the $\gamma$-ray luminosity as a function of the proton spectral index based on the results obtained from the joint likelihood analysis with $1 / d^{2}$ weighting shown in blue compared to the limit obtained from the closest single source SN $2011 \mathrm{ht}$ in green. Right: $95 \%$ CL upper limit on the ratio of $\gamma$-ray and optical luminosity $L_{\gamma} / L_{R}$ as a function of $\Gamma_{\mathrm{p}}$ assuming a proportionality between optical and $\gamma$-ray flux shown in blue compared to the limit obtained from a single source analysis of SN 2010jl considering a one year time window (in green). The results of the analysis with an extended time window of 4.5 years for SN $2010 \mathrm{jl}$ are shown in dashed green.

Program of the Planning and Budgeting Committee and The Israel Science Foundation. A.G.-Y. is supported by the EU/FP7 via ERC grant No. 307260, the Quantum Universe I-Core program by the Israeli Committee for Planning and Budgeting and the ISF, Minerva and ISF grants, WIS-UK "Making Connections", and Kimmel and ARCHES awards.

\section{APPENDIX A SN CATALOG}

The following table contains all $\mathrm{SNe}$ included in this analysis. The column definition is similar to Table 1 .

\begin{tabular}{|c|c|c|c|c|c|c|}
\hline Name & $\begin{array}{c}\text { R. } \\
\text { A. }\left({ }^{\circ}\right)^{\star}\end{array}$ & $\begin{array}{l}\text { Decl. } \\
\left({ }^{\circ}\right)^{\star}\end{array}$ & Date & $z$ & $m$ & $\begin{array}{l}\text { TS ( } p \text { - } \\
\text { value) }\end{array}$ \\
\hline SN 2008gm & 348.55 & -2.78 & $\begin{array}{c}2008 \\
\text { Oct } 22^{\dagger}\end{array}$ & 0.012 & $17.00^{*}$ & $\begin{array}{c}3.2 \\
(0.169)\end{array}$ \\
\hline CSS081201_103354-032125 & 158.47 & -3.36 & $\begin{array}{c}2008 \\
\text { Dec } 01^{\text {* }}\end{array}$ & 0.060 & $18.30^{*}$ & $\begin{array}{c}0.0 \\
(0.572)\end{array}$ \\
\hline $\begin{array}{l}\text { CSS } 080701 \_234413+ \\
075224\end{array}$ & 356.05 & 7.87 & $\begin{array}{l}2008 \\
\text { Dec }\end{array}$ & 0.069 & 18.50 & $\begin{array}{l}0.0 \\
(0.572)\end{array}$ \\
\hline SN $2008 \mathrm{ja}$ & & & 30 & & & \\
\hline SN 2008ip & 194.46 & 36.38 & $\begin{array}{c}2008 \\
\text { Dec } 31^{\dagger}\end{array}$ & 0.015 & $15.70^{*}$ & $\begin{array}{c}0.0 \\
(0.572)\end{array}$ \\
\hline SN 2009au & 194.94 & -29.60 & $\begin{array}{c}2009 \\
\operatorname{Mar} 11^{\dagger}\end{array}$ & 0.009 & $16.40^{*}$ & $\begin{array}{c}0.0 \\
(0.572)\end{array}$ \\
\hline $\begin{array}{l}\text { CSS } 080928 \_160837+ \\
041626\end{array}$ & 242.16 & 4.27 & $\begin{array}{l}2009 \\
\text { Mar }\end{array}$ & 0.041 & 17.60 & $\begin{array}{l}0.3 \\
(0.458)\end{array}$ \\
\hline SN 2008iy & & & 21 & & & \\
\hline SN $2009 \mathrm{cw}$ & 226.26 & 48.67 & $\begin{array}{c}2009 \\
\operatorname{Mar} 28^{\dagger}\end{array}$ & 0.150 & $20.30^{\ddagger}$ & $\begin{array}{c}0.0 \\
(0.572)\end{array}$ \\
\hline SN 2009eo & 224.53 & 2.43 & $\begin{array}{c}2009 \\
\text { May } \\
13^{\dagger}\end{array}$ & 0.044 & $18.10^{*}$ & $\begin{array}{c}0.0 \\
(0.572)\end{array}$ \\
\hline SN 2009fs & 274.80 & 42.81 & $\begin{array}{c}2009 \\
\text { Jun } 01^{\dagger}\end{array}$ & 0.054 & $17.00^{\ddagger}$ & $\begin{array}{c}3.5 \\
(0.154)\end{array}$ \\
\hline PTF 09ij & 218.06 & 54.86 & $\begin{array}{c}2009 \\
\text { Jun } 03\end{array}$ & 0.124 & 20.30 & $\begin{array}{c}0.0 \\
(0.572)\end{array}$ \\
\hline PTF 09ge & 224.26 & 49.61 & $\begin{array}{c}2009 \\
\text { Jun } 06\end{array}$ & 0.064 & 17.90 & $\begin{array}{c}3.3 \\
(0.165)\end{array}$ \\
\hline PTF 09tm & 206.73 & 61.55 & $\begin{array}{c}2009 \\
\text { Jun } 25\end{array}$ & 0.034 & 16.80 & $\begin{array}{c}0.0 \\
(0.572)\end{array}$ \\
\hline PTF 09uj & 215.05 & 53.56 & $\begin{array}{c}2009 \\
\text { Jun } 26\end{array}$ & 0.066 & 18.20 & $\begin{array}{c}0.0 \\
(0.572)\end{array}$ \\
\hline PTF 09uy & 190.98 & 74.69 & & 0.313 & 19.40 & \\
\hline
\end{tabular}

\begin{tabular}{|c|c|c|c|c|c|c|}
\hline \multicolumn{7}{|c|}{ (Continued) } \\
\hline Name & $\begin{array}{l}\text { R. } \\
\text { A. }\left({ }^{\circ}\right)^{*}\end{array}$ & $\begin{array}{l}\text { Decl. } \\
\left({ }^{\circ}\right)^{\star}\end{array}$ & Date & $z$ & $m$ & $\begin{array}{l}\text { TS }(p- \\
\text { value })\end{array}$ \\
\hline & & & $\begin{array}{c}2009 \\
\text { Jul } 03\end{array}$ & & & $\begin{array}{c}0.0 \\
(0.572)\end{array}$ \\
\hline PTF 09bcl & 271.61 & 17.86 & $\begin{array}{c}2009 \\
\text { Jul } 19^{\dagger}\end{array}$ & 0.062 & $20.87^{*}$ & $\begin{array}{c}0.0 \\
(0.572)\end{array}$ \\
\hline PTF 10ujc & 353.63 & 22.35 & $\begin{array}{c}2009 \\
\text { Aug } 05\end{array}$ & 0.032 & 16.20 & $\begin{array}{c}0.0 \\
(0.572)\end{array}$ \\
\hline PTF 09drs & 226.63 & 60.59 & $\begin{array}{c}2009 \\
\text { Aug } 15\end{array}$ & 0.045 & 18.50 & $\begin{array}{c}0.0 \\
(0.561)\end{array}$ \\
\hline $\begin{array}{c}\text { CSS090925_001259+ } \\
144121\end{array}$ & 3.25 & 14.69 & $\begin{array}{c}2009 \\
\text { Sep } 25^{\dagger}\end{array}$ & 0.090 & $18.80^{\frac{7}{7}}$ & $\begin{array}{c}0.0 \\
(0.568)\end{array}$ \\
\hline SN 2009ma & 127.24 & 0.59 & $\begin{array}{c}2009 \\
\text { Oct } 17^{\dagger}\end{array}$ & 0.089 & $18.20^{\frac{*}{7}}$ & $\begin{array}{c}0.0 \\
(0.572)\end{array}$ \\
\hline $\begin{array}{l}\text { CSS091018_091109+ } \\
195945\end{array}$ & 137.79 & 20.00 & $\begin{array}{l}2009 \\
\text { Oct }\end{array}$ & 0.150 & $19.00^{*}$ & $\begin{array}{l}0.0 \\
(0.572)\end{array}$ \\
\hline SN 2009mb & & & $18^{\dagger}$ & & & \\
\hline SN 2009kn & 122.43 & -17.75 & $\begin{array}{c}2009 \\
\text { Oct } 26^{\dagger}\end{array}$ & 0.016 & $16.60^{*}$ & $\begin{array}{c}0.0 \\
(0.572)\end{array}$ \\
\hline $\mathrm{SN} 2009 \mathrm{kr}$ & 78.01 & -15.70 & $\begin{array}{l}2009 \\
\text { Nov } \\
06^{\dagger}\end{array}$ & 0.006 & $16.00^{\frac{7}{4}}$ & $\begin{array}{c}4.7 \\
(0.104)\end{array}$ \\
\hline SN $2009 \mathrm{~nm}$ & 151.35 & 51.28 & $\begin{array}{l}2009 \\
\text { Nov } \\
20^{\dagger}\end{array}$ & 0.210 & $18.80^{\frac{7}{7}}$ & $\begin{array}{c}0.0 \\
(0.572)\end{array}$ \\
\hline $\begin{array}{l}\text { CSS091217_110637 + } \\
341952\end{array}$ & 166.65 & 34.33 & $\begin{array}{l}2009 \\
\text { Dec }\end{array}$ & $?$ & $18.70^{\ddagger}$ & $\begin{array}{l}0.0 \\
(0.572)\end{array}$ \\
\hline SN 2009nj & & & $17^{\dagger}$ & & & \\
\hline $\begin{array}{l}\text { CSS091218_104011 + } \\
223735\end{array}$ & 160.05 & 22.63 & $\begin{array}{l}2009 \\
\text { Dec }\end{array}$ & 0.140 & $19.40^{\frac{*}{3}}$ & $\begin{array}{l}0.0 \\
(0.572)\end{array}$ \\
\hline SN 2009nw & & & $18^{\dagger}$ & & & \\
\hline PTF 10dk & 77.09 & 0.21 & $\begin{array}{c}2009 \\
\text { Dec } 18^{\dagger}\end{array}$ & 0.074 & $20.14^{\ddagger}$ & $\begin{array}{c}0.0 \\
(0.572)\end{array}$ \\
\hline PTF 10u & 152.49 & 46.01 & $\begin{array}{c}2010 \\
\text { Jan } 05\end{array}$ & 0.150 & 19.80 & $\begin{array}{c}0.0 \\
(0.572)\end{array}$ \\
\hline PTF 11ner & 125.58 & 72.83 & $\begin{array}{c}2010 \\
\operatorname{Jan} 11^{\dagger}\end{array}$ & 0.117 & $20.94^{\ddagger}$ & $\begin{array}{c}0.0 \\
(0.572)\end{array}$ \\
\hline $\begin{array}{l}\text { PTF 10ts } \\
\text { SN 2009nn }\end{array}$ & 188.49 & 13.92 & $\begin{array}{r}2010 \\
\text { Jan } \\
12\end{array}$ & 0.046 & 17.66 & $\begin{array}{l}7.9 \\
(0.033)\end{array}$ \\
\hline $\begin{array}{l}\text { CSS100113_032138 + } \\
263650\end{array}$ & 50.41 & 26.61 & $\begin{array}{r}2010 \\
\text { Jan }\end{array}$ & 0.060 & $18.80^{\frac{\$}{4}}$ & $\begin{array}{l}0.1 \\
(0.517)\end{array}$ \\
\hline SN 2010M & & & $13^{\dagger}$ & & & \\
\hline PTF 10cwl & 189.09 & 7.79 & & 0.085 & $19.00^{\frac{*}{4}}$ & \\
\hline
\end{tabular}


(Continued)

\begin{tabular}{|c|c|c|c|c|c|c|}
\hline Name & $\begin{array}{c}\text { R. } \\
\text { A. }\left({ }^{\circ}\right)^{\star}\end{array}$ & $\begin{array}{l}\text { Decl. } \\
\left({ }^{\circ}\right)^{\star}\end{array}$ & Date & $z$ & $m$ & $\begin{array}{l}\text { TS }(p- \\
\text { value })\end{array}$ \\
\hline & & & $\begin{array}{c}2010 \\
\operatorname{Mar} 13^{\dagger}\end{array}$ & & & $\begin{array}{c}0.0 \\
(0.572)\end{array}$ \\
\hline SN 2010al & 123.57 & 18.44 & $\begin{array}{c}2010 \\
\operatorname{Mar} 13^{\dagger}\end{array}$ & 0.017 & $17.80^{\ddagger}$ & $\begin{array}{c}9.7 \\
(0.023)\end{array}$ \\
\hline PTF 10cwx & 188.32 & -0.05 & $\begin{array}{c}2010 \\
\text { Mar } 19\end{array}$ & 0.073 & 18.50 & $\begin{array}{c}2.3 \\
(0.228)\end{array}$ \\
\hline PTF 10fei & 227.07 & 53.59 & $\begin{array}{c}2010 \\
\text { Apr } 04\end{array}$ & 0.090 & 18.55 & $\begin{array}{c}0.0 \\
(0.572)\end{array}$ \\
\hline PTF 10fel & 246.88 & 51.36 & $\begin{array}{c}2010 \\
\text { Apr } 04\end{array}$ & 0.234 & 19.70 & $\begin{array}{c}11.1 \\
(0.016)\end{array}$ \\
\hline PTF 10ewc & 210.50 & 33.84 & $\begin{array}{c}2010 \\
\text { Apr } 15\end{array}$ & 0.055 & 18.40 & $\begin{array}{c}0.3 \\
(0.476)\end{array}$ \\
\hline PTF 10fou & 208.94 & 29.88 & $\begin{array}{c}2010 \\
\text { Apr } 17\end{array}$ & 0.043 & 20.00 & $\begin{array}{c}0.2 \\
(0.489)\end{array}$ \\
\hline PTF 10flx & 251.74 & 64.45 & $\begin{array}{c}2010 \\
\text { Apr } 17\end{array}$ & 0.067 & 18.80 & $\begin{array}{c}11.6 \\
(0.015)\end{array}$ \\
\hline SN 2010bt & 192.08 & -34.95 & $\begin{array}{c}2010 \\
\text { Apr } 17^{\dagger}\end{array}$ & 0.016 & $15.80^{*}$ & $\begin{array}{c}14.4 \\
(0.0065)\end{array}$ \\
\hline $\begin{array}{l}\text { PTF 10fjh } \\
\text { SN 2010bq }\end{array}$ & 251.73 & 34.16 & $\begin{array}{c}2010 \\
\text { Apr } \\
25\end{array}$ & 0.032 & 17.20 & $\begin{array}{l}0.0 \\
(0.572)\end{array}$ \\
\hline PTF 10gvd & 253.26 & 67.00 & $\begin{array}{c}2010 \\
\text { May } 02\end{array}$ & 0.070 & 19.20 & $\begin{array}{c}2.8 \\
(0.196)\end{array}$ \\
\hline PTF 10hcr & 183.00 & 38.53 & $\begin{array}{c}2010 \\
\text { May } \\
06^{\dagger}\end{array}$ & 0.037 & $20.06^{*}$ & $\begin{array}{c}1.0 \\
(0.359)\end{array}$ \\
\hline PTF 10hbf & 193.19 & -6.92 & $\begin{array}{c}2010 \\
\text { May } 07\end{array}$ & 0.042 & 18.80 & $\begin{array}{c}0.6 \\
(0.407)\end{array}$ \\
\hline PTF 10hif & 257.45 & 27.26 & $\begin{array}{c}2010 \\
\text { May } 12\end{array}$ & 0.141 & 18.00 & $\begin{array}{c}0.0 \\
(0.572)\end{array}$ \\
\hline PTF 10gvf & 168.44 & 53.63 & $\begin{array}{c}2010 \\
\text { May } 14\end{array}$ & 0.080 & 19.00 & $\begin{array}{c}0.0 \\
(0.572)\end{array}$ \\
\hline PTF 10hSN & 244.40 & 5.04 & $\begin{array}{c}2010 \\
\text { Jun } 01\end{array}$ & 0.164 & 19.00 & $\begin{array}{c}0.0 \\
(0.572)\end{array}$ \\
\hline PTF 10jop & 322.38 & 2.88 & $\begin{array}{c}2010 \\
\text { Jun } 11\end{array}$ & 0.089 & 18.60 & $\begin{array}{c}0.0 \\
(0.572)\end{array}$ \\
\hline PTF 10ngx & 186.80 & 15.98 & $\begin{array}{c}2010 \\
\text { Jul } 03\end{array}$ & 0.067 & 19.40 & $\begin{array}{c}0.0 \\
(0.572)\end{array}$ \\
\hline PTF 10ndr & 224.95 & 65.00 & $\begin{array}{c}2010 \\
\text { Jul } 26\end{array}$ & 0.075 & 19.60 & $\begin{array}{c}0.0 \\
(0.572)\end{array}$ \\
\hline PTF 10qaf & 353.93 & 10.78 & $\begin{array}{c}2010 \\
\text { Jul } 31\end{array}$ & 0.284 & 19.00 & $\begin{array}{c}7.5 \\
(0.036)\end{array}$ \\
\hline SN 2010hd & 340.47 & -46.10 & $\begin{array}{c}2010 \\
\text { Aug } \\
07^{\dagger}\end{array}$ & 0.033 & $17.60^{\ddagger}$ & $\begin{array}{c}8.8 \\
(0.028)\end{array}$ \\
\hline PS1-1000789 & 310.69 & 15.51 & $\begin{array}{c}2010 \\
\text { Aug } \\
15^{\dagger}\end{array}$ & 0.200 & $17.30^{*}$ & $\begin{array}{c}0.0 \\
(0.572)\end{array}$ \\
\hline PTF 10oug & 260.19 & 29.07 & $\begin{array}{c}2010 \\
\text { Aug } 20\end{array}$ & 0.150 & 19.20 & $\begin{array}{c}0.0 \\
(0.572)\end{array}$ \\
\hline PTF 10scc & 352.04 & 28.64 & $\begin{array}{c}2010 \\
\text { Aug } 20\end{array}$ & 0.242 & 18.90 & $\begin{array}{c}0.0 \\
(0.572)\end{array}$ \\
\hline PTF 10qwu & 252.79 & 28.30 & $\begin{array}{c}2010 \\
\text { Aug } 20\end{array}$ & 0.226 & 19.40 & $\begin{array}{c}0.1 \\
(0.541)\end{array}$ \\
\hline PTF 10tjr & 220.38 & 23.01 & $\begin{array}{c}2010 \\
\text { Aug } \\
23^{\dagger}\end{array}$ & 0.078 & $17.73^{*}$ & $\begin{array}{c}0.0 \\
(0.572)\end{array}$ \\
\hline PTF 10tpz & 329.63 & -15.55 & $\begin{array}{c}2010 \\
\text { Aug } \\
28^{\dagger}\end{array}$ & 0.040 & $17.06^{*}$ & $\begin{array}{c}0.0 \\
(0.572)\end{array}$ \\
\hline $\begin{array}{l}\text { PTF 10tel } \\
\text { SN 2010mc }\end{array}$ & 260.38 & 48.13 & $\begin{array}{c}2010 \\
\text { Sep } \\
04\end{array}$ & 0.035 & 17.50 & $\begin{array}{l}0.0 \\
(0.572)\end{array}$ \\
\hline PTF 10ttp & 341.92 & -10.04 & $\begin{array}{c}2010 \\
\text { Sep } 09\end{array}$ & 0.179 & 19.50 & $\begin{array}{c}0.0 \\
(0.572)\end{array}$ \\
\hline $\begin{array}{l}\text { CSS100910_001539+ } \\
271250\end{array}$ & 3.91 & 27.21 & $\begin{array}{c}2010 \\
\text { Sep } 10^{\dagger}\end{array}$ & 0.024 & $18.10^{\ddagger}$ & $\begin{array}{c}0.0 \\
(0.572)\end{array}$ \\
\hline $\begin{array}{l}\text { PTF 10viv } \\
\text { SN 2010jg }\end{array}$ & 331.11 & -7.98 & $\begin{array}{r}2010 \\
\text { Sep } \\
12^{\dagger}\end{array}$ & 0.060 & $20.13^{*}$ & $\begin{array}{l}0.0 \\
(0.572)\end{array}$ \\
\hline
\end{tabular}

(Continued)

\begin{tabular}{|c|c|c|c|c|c|c|}
\hline Name & $\begin{array}{c}\text { R. } \\
\text { A. }\left({ }^{\circ}\right)^{\star}\end{array}$ & $\begin{array}{l}\text { Decl. } \\
\left({ }^{\circ}\right)^{\star}\end{array}$ & Date & $z$ & $m$ & $\begin{array}{l}\text { TS }(p- \\
\text { value })\end{array}$ \\
\hline PTF 10uls & 20.34 & 4.89 & $\begin{array}{c}2010 \\
\text { Sep } 19\end{array}$ & 0.044 & 18.60 & $\begin{array}{c}1.4 \\
(0.322)\end{array}$ \\
\hline PTF 10xzs & 120.60 & 67.42 & $\begin{array}{c}2010 \\
\text { Sep } 22^{\dagger}\end{array}$ & 0.036 & $19.33^{\frac{4}{+}}$ & $\begin{array}{c}8.2 \\
(0.031)\end{array}$ \\
\hline PTF 10wop & 327.65 & -6.77 & $\begin{array}{c}2010 \\
\text { Sep } 23^{\dagger}\end{array}$ & 0.090 & $19.55^{\frac{\hbar}{*}}$ & $\begin{array}{c}0.0 \\
(0.572)\end{array}$ \\
\hline PTF 10xif & 48.11 & -9.81 & $\begin{array}{c}2010 \\
\text { Sep } 27^{\dagger}\end{array}$ & 0.029 & $18.42^{\text {के }}$ & $\begin{array}{c}0.0 \\
(0.572)\end{array}$ \\
\hline PTF 10vag & 326.83 & 18.13 & $\begin{array}{c}2010 \\
\text { Sep } 29\end{array}$ & 0.052 & 18.50 & $\begin{array}{c}0.0 \\
(0.572)\end{array}$ \\
\hline PTF 10xgo & 328.99 & 1.32 & $\begin{array}{c}2010 \\
\text { Oct } 03^{\dagger}\end{array}$ & 0.034 & $19.25^{\frac{\hbar}{2}}$ & $\begin{array}{c}2.8 \\
(0.193)\end{array}$ \\
\hline CSS121009_025917-141610 & 44.82 & -14.27 & $\begin{array}{c}2010 \\
\text { Oct } 09^{\dagger}\end{array}$ & 0.080 & $19.20^{\frac{3}{2}}$ & $\begin{array}{c}0.0 \\
(0.572)\end{array}$ \\
\hline PTF 10tyd & 257.33 & 27.82 & $\begin{array}{c}2010 \\
\text { Oct } 09\end{array}$ & 0.063 & 19.00 & $\begin{array}{c}0.0 \\
(0.572)\end{array}$ \\
\hline PTF 12kph & 24.82 & -7.56 & $\begin{array}{c}2010 \\
\text { Oct } 11^{\dagger}\end{array}$ & 0.059 & $18.84^{\ddagger}$ & $\begin{array}{c}6.1 \\
(0.063)\end{array}$ \\
\hline PTF 10uiz & 258.63 & 21.43 & $\begin{array}{c}2010 \\
\text { Oct } 19\end{array}$ & 0.114 & 18.40 & $\begin{array}{c}0.0 \\
(0.572)\end{array}$ \\
\hline PTF 10wmk & 132.04 & 55.83 & $\begin{array}{c}2010 \\
\text { Oct } 29\end{array}$ & 0.137 & 19.51 & $\begin{array}{c}0.0 \\
(0.572)\end{array}$ \\
\hline PTF 10yzt & 2.96 & 26.69 & $\begin{array}{c}2010 \\
\text { Oct } 29^{\dagger}\end{array}$ & 0.076 & $18.58^{\circ}$ & $\begin{array}{c}0.0 \\
(0.572)\end{array}$ \\
\hline $\begin{array}{l}\text { CSS101030_230944 + } \\
054156\end{array}$ & 347.43 & 5.70 & $\begin{array}{r}2010 \\
\text { Oct }\end{array}$ & 0.042 & $16.50^{\frac{1}{5}}$ & $\begin{array}{l}0.0 \\
(0.572)\end{array}$ \\
\hline $\begin{array}{l}\text { SN 2010jy } \\
\text { PTF 10aaes }\end{array}$ & 31.79 & 16.21 & $\begin{array}{c}30^{\dagger} \\
2010 \\
\operatorname{Oct} 30^{\dagger}\end{array}$ & 0.037 & $19.50^{*}$ & $\begin{array}{c}0.0 \\
(0.572)\end{array}$ \\
\hline SN 2010jk & 18.15 & 15.47 & $\begin{array}{c}2010 \\
\text { Oct } 31^{\dagger}\end{array}$ & 0.280 & $20.20^{\frac{7}{2}}$ & $\begin{array}{c}(0.5 / 2) \\
3.6 \\
(0.153)\end{array}$ \\
\hline PTF 10acfd & 147.91 & 1.52 & $\begin{array}{c}2010 \\
\text { Nov } \\
03^{\dagger}\end{array}$ & 0.192 & $20.34^{\frac{3}{2}}$ & $\begin{array}{c}0.0 \\
(0.572)\end{array}$ \\
\hline SN 2010lx & 71.19 & -22.21 & $\begin{array}{c}2010 \\
\text { Nov } \\
03^{\dagger}\end{array}$ & 0.100 & $18.70^{\frac{3}{2}}$ & $\begin{array}{c}0.0 \\
(0.572)\end{array}$ \\
\hline SN 2010js & 124.21 & 60.50 & $\begin{array}{c}2010 \\
\text { Nov } \\
07^{\dagger}\end{array}$ & 0.039 & $18.10^{\frac{3}{5}}$ & $\begin{array}{c}1.9 \\
(0.262)\end{array}$ \\
\hline PTF 10yyc & 69.82 & -0.35 & $\begin{array}{c}2010 \\
\text { Nov } 08\end{array}$ & 0.214 & 17.66 & $\begin{array}{c}0.9 \\
(0.367)\end{array}$ \\
\hline PTF 10weh & 261.71 & 58.85 & $\begin{array}{c}2010 \\
\text { Nov } 08\end{array}$ & 0.138 & 18.30 & $\begin{array}{c}6.6 \\
(0.048)\end{array}$ \\
\hline $\begin{array}{l}\text { CSS101110_082047+ } \\
355337 \\
2010 \mathrm{~kb}\end{array}$ & 125.20 & 35.89 & $\begin{array}{c}2010 \\
\text { Nov } \\
10^{\dagger}\end{array}$ & 0.075 & $18.20^{\frac{5}{5}}$ & $\begin{array}{l}6.2 \\
(0.059)\end{array}$ \\
\hline $\begin{array}{l}\text { PTF 10aazn } \\
\text { SN 2010jj }\end{array}$ & 31.72 & 44.57 & $\begin{array}{c}2010 \\
\text { Nov } \\
13^{\dagger}\end{array}$ & 0.016 & $16.52^{\frac{\hbar}{4}}$ & $\begin{array}{l}5.4 \\
(0.079)\end{array}$ \\
\hline $\begin{array}{l}\text { PTF 10aaxf } \\
\text { SN 2010jl }\end{array}$ & 145.72 & 9.50 & $\begin{array}{c}2010 \\
\text { Nov } \\
18\end{array}$ & 0.011 & 13.20 & $\begin{array}{l}7.1 \\
(0.039)\end{array}$ \\
\hline PTF 10abcl & 348.90 & 22.81 & $\begin{array}{c}2010 \\
\text { Nov } \\
19^{\dagger}\end{array}$ & 0.061 & $18.95^{\circ}$ & $\begin{array}{c}3.0 \\
(0.181)\end{array}$ \\
\hline $\begin{array}{l}\text { PTF 10aaxi } \\
\text { SN 2010jp }\end{array}$ & 94.13 & -21.41 & $\begin{array}{c}2010 \\
\text { Nov } \\
23\end{array}$ & 0.010 & 18.00 & $\begin{array}{l}0.0 \\
(0.572)\end{array}$ \\
\hline PTF 10yni & 2.71 & 14.18 & $\begin{array}{c}2010 \\
\text { Nov } 28\end{array}$ & 0.169 & 18.90 & $\begin{array}{c}7.2 \\
(0.039)\end{array}$ \\
\hline PTF 10abui & 93.08 & -22.77 & $\begin{array}{c}2010 \\
\text { Dec } 08\end{array}$ & 0.052 & 18.60 & $\begin{array}{c}0.0 \\
(0.572)\end{array}$ \\
\hline PTF 10abyy & 79.17 & 6.80 & $\begin{array}{c}2010 \\
\text { Dec } 08^{\dagger}\end{array}$ & 0.030 & $18.66^{\frac{5}{2}}$ & $\begin{array}{c}0.2 \\
(0.503)\end{array}$ \\
\hline PTF 10achk & 46.49 & -10.52 & $\begin{array}{c}2010 \\
\text { Dec } 28\end{array}$ & 0.033 & 16.90 & $\begin{array}{c}0.0 \\
(0.572)\end{array}$ \\
\hline SN 2011A & 195.25 & -14.53 & $\begin{array}{c}2011 \\
\text { Jan } 02^{\dagger}\end{array}$ & 0.009 & $16.90^{\frac{*}{5}}$ & $\begin{array}{c}0.0 \\
(0.572)\end{array}$ \\
\hline SN 2011P & 36.44 & 16.22 & & 0.080 & $18.60^{\ddagger}$ & \\
\hline
\end{tabular}




\begin{tabular}{|c|c|c|c|c|c|c|}
\hline \multicolumn{7}{|c|}{ (Continued) } \\
\hline \multirow[t]{3}{*}{ Name } & $\begin{array}{c}\text { R. } \\
\text { A. }\left({ }^{\circ}\right)^{\star}\end{array}$ & $\begin{array}{l}\text { Decl. } \\
\left({ }^{\circ}\right)^{\star}\end{array}$ & Date & $z$ & $m$ & $\begin{array}{l}\text { TS }(p- \\
\text { value })\end{array}$ \\
\hline & & & 2011 & & & 10.8 \\
\hline & & & Jan $05^{\dagger}$ & & & $(0.016)$ \\
\hline \multirow[t]{2}{*}{ SN 2011af } & 36.48 & 10.39 & 2011 & 0.064 & $16.70^{\text {米 }}$ & 0.0 \\
\hline & & & Jan $11^{\dagger}$ & & & $(0.572)$ \\
\hline \multirow[t]{2}{*}{ SN 2011S } & 138.48 & -17.01 & 2011 & 0.060 & $17.60^{\ddagger}$ & 0.0 \\
\hline & & & Jan $14^{\dagger}$ & & & $(0.572)$ \\
\hline \multirow[t]{2}{*}{ PTF 10acsq } & 120.39 & 46.76 & 2011 & 0.173 & 19.00 & 0.0 \\
\hline & & & Jan 27 & & & $(0.572)$ \\
\hline \multirow[t]{2}{*}{ SN 2011ap } & 272.62 & 31.01 & 2011 & 0.024 & $18.30^{*}$ & 0.0 \\
\hline & & & Feb $21^{\dagger}$ & & & $(0.572)$ \\
\hline \multirow[t]{2}{*}{ SN 2011an } & 119.85 & 16.42 & 2011 & 0.016 & $18.40^{\ddagger}$ & 0.7 \\
\hline & & & Mar $01^{\dagger}$ & & & $(0.398)$ \\
\hline \multirow[t]{2}{*}{ SN 2011cc } & 248.46 & 39.26 & 2011 & 0.032 & $17.70^{\ddagger}$ & 0.0 \\
\hline & & & $\operatorname{Mar} 17^{\dagger}$ & & & $(0.572)$ \\
\hline \multirow[t]{2}{*}{ PS1-11xn } & 221.91 & 51.68 & 2011 & 0.040 & $18.60^{*}$ & 0.0 \\
\hline & & & Apr $26^{\dagger}$ & & & $(0.572)$ \\
\hline \multirow[t]{2}{*}{ SN $2011 \mathrm{cp}$} & 118.14 & 21.89 & 2011 & 0.390 & $19.50^{\ddagger}$ & 5.4 \\
\hline & & & Apr $26^{\dagger}$ & & & $(0.081)$ \\
\hline CSS110501_094825 + & 147.10 & 20.73 & 2011 & 0.040 & $18.40^{\ddagger}$ & 0.0 \\
\hline 204333 & & & May & & & $(0.572)$ \\
\hline & & & $01^{\dagger}$ & & & \\
\hline PTF $11 \mathrm{csc}$ & 224.68 & 36.60 & 2011 & 0.117 & 20.60 & 0.0 \\
\hline & & & May 02 & & & $(0.572)$ \\
\hline PTF 11dsb & 244.65 & 32.70 & 2011 & 0.190 & 20.10 & 0.0 \\
\hline & & & May 15 & & & $(0.572)$ \\
\hline SN 2011eu & 212.31 & -1.18 & 2011 & 0.110 & $18.50^{\ddagger}$ & 0.0 \\
\hline & & & Jun $06^{\dagger}$ & & & $(0.572)$ \\
\hline PTF 11 fuu & 325.12 & 6.33 & 2011 & 0.097 & 18.50 & 0.0 \\
\hline & & & Jun 09 & & & $(0.572)$ \\
\hline PTF $11 \mathrm{fss}$ & 323.47 & 1.84 & 2011 & 0.125 & $19.42^{*}$ & 0.0 \\
\hline & & & Jun $11^{\dagger}$ & & & $(0.572)$ \\
\hline CSS110623_131919-045106 & 199.83 & -4.85 & 2011 & 0.070 & $18.40^{\ddagger}$ & 0.0 \\
\hline & & & Jun $23^{\dagger}$ & & & $(0.572)$ \\
\hline PTF 11gtr & 258.01 & 23.38 & 2011 & 0.029 & $20.94^{*}$ & 0.0 \\
\hline & & & Jun $25^{\dagger}$ & & & $(0.572)$ \\
\hline PTF $11 \mathrm{hzx}$ & 327.67 & 18.11 & 2011 & 0.229 & 18.90 & 0.0 \\
\hline & & & Jul 24 & & & $(0.572)$ \\
\hline PTF 11iqb & 8.52 & -9.70 & 2011 & 0.013 & 15.20 & 0.3 \\
\hline & & & Aug 06 & & & $(0.469)$ \\
\hline PTF $11 \mathrm{fzz}$ & 167.69 & 54.11 & 2011 & 0.082 & 17.40 & 0.3 \\
\hline & & & Aug 15 & & & $(0.479)$ \\
\hline SN 2011fh & 194.06 & -29.50 & 2011 & 0.008 & $14.50^{\ddagger}$ & 1.9 \\
\hline & & & Aug & & & $(0.262)$ \\
\hline & & & $24^{\dagger}$ & & & \\
\hline PTF 11pab & 44.63 & 6.31 & 2011 & 0.022 & $21.08^{\ddagger}$ & 6.1 \\
\hline & & & Aug & & & $(0.063)$ \\
\hline & & & $30^{\dagger}$ & & & \\
\hline SN 2011fx & 4.50 & 24.56 & 2011 & 0.019 & $17.60^{\ddagger}$ & 0.5 \\
\hline & & & Aug & & & $(0.428)$ \\
\hline & & & $30^{\dagger}$ & & & \\
\hline SN $2011 \mathrm{fr}$ & 22.44 & 18.89 & 2011 & 0.060 & $18.80^{\ddagger}$ & 0.0 \\
\hline & & & Sep $01^{\dagger}$ & & & $(0.572)$ \\
\hline PTF 11mpg & 334.40 & 0.61 & 2011 & 0.093 & 19.18 & 0.0 \\
\hline & & & Sep 19 & & & $(0.572)$ \\
\hline PTF 11oey & 352.73 & 23.18 & 2011 & 0.061 & $20.17^{*}$ & 0.0 \\
\hline & & & Sep $21^{\dagger}$ & & & $(0.572)$ \\
\hline PTF $11 \mathrm{mtq}$ & 270.08 & 28.70 & 2011 & 0.073 & $19.35^{*}$ & 1.6 \\
\hline & & & Sep $22^{\dagger}$ & & & $(0.302)$ \\
\hline PTF 11msk & 325.91 & -1.69 & 2011 & 0.070 & 19.10 & 2.2 \\
\hline & & & Oct 04 & & & $(0.238)$ \\
\hline PTF 11pdt & 44.63 & 6.31 & 2011 & 0.022 & $20.00^{\ddagger}$ & 8.9 \\
\hline & & & Oct $19^{\dagger}$ & & & $(0.028)$ \\
\hline PSNJ $10081059+5150570$ & 152.04 & 51.85 & 2011 & 0.004 & 14.50 & 0.0 \\
\hline SN 2011ht & & & Oct & & & $(0.572)$ \\
\hline & & & 29 & & & \\
\hline PTF 11qnf & 86.23 & 69.15 & 2011 & 0.014 & $19.80^{\ddagger}$ & 1.4 \\
\hline & & & Nov & & & $(0.320)$ \\
\hline & & & $01^{\dagger}$ & & & \\
\hline SN $2011 \mathrm{ib}$ & 176.16 & 35.97 & & 0.037 & $16.80^{\ddagger}$ & $\begin{array}{c}0.0 \\
(0.572)\end{array}$ \\
\hline
\end{tabular}

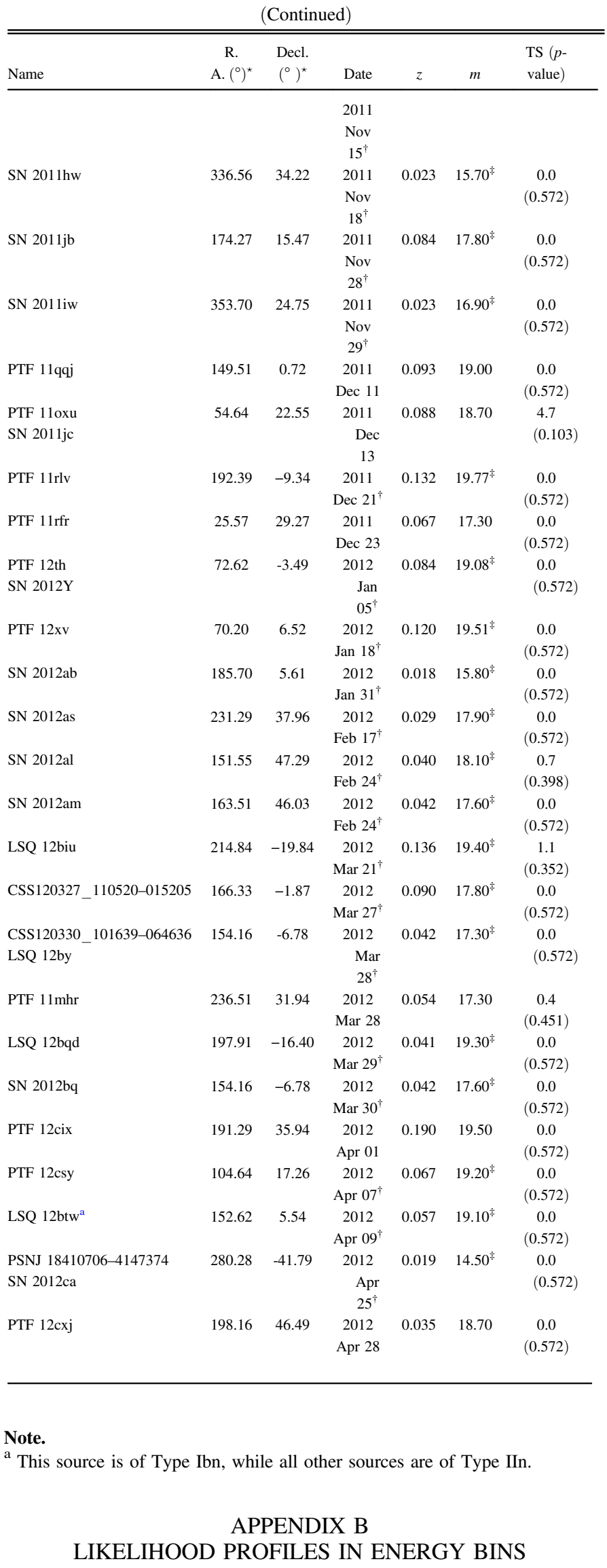

Figures 10 and 11 show the likelihood profiles in energy bins for $\Delta T=1$ year and the $95 \%$ CL upper limit for the three time 

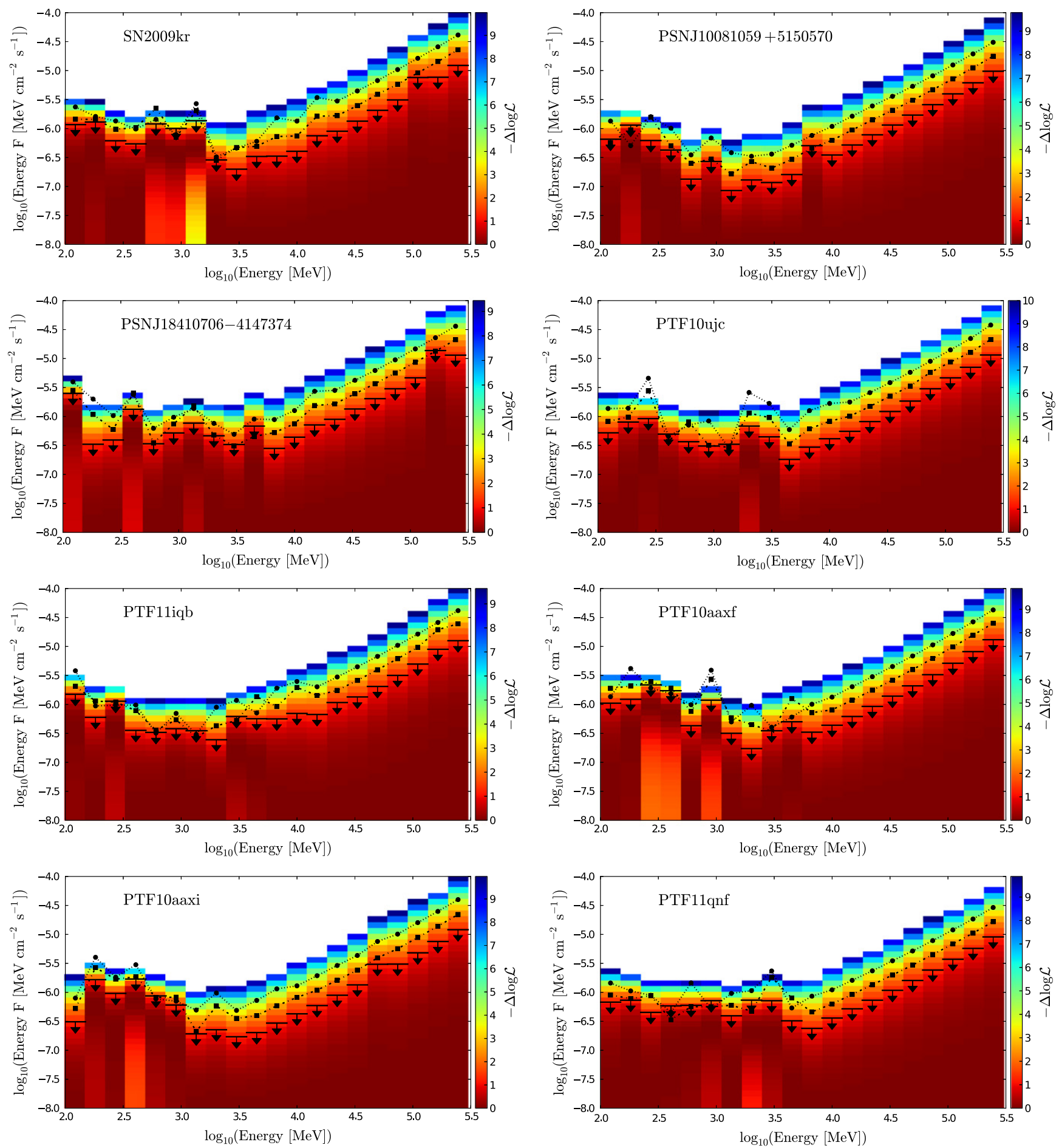

Figure 10. Similar to Figure 4. Colors represent the likelihood profile for each energy for $\Delta T=1$ year. The black arrows indicate the $95 \%$ CL upper limits for $\Delta T=1$ year, while the dotted-dashed and dotted lines represent the $95 \%$ CL upper limit for $\Delta T=6$ months and $\Delta T=3$ months, respectively. 

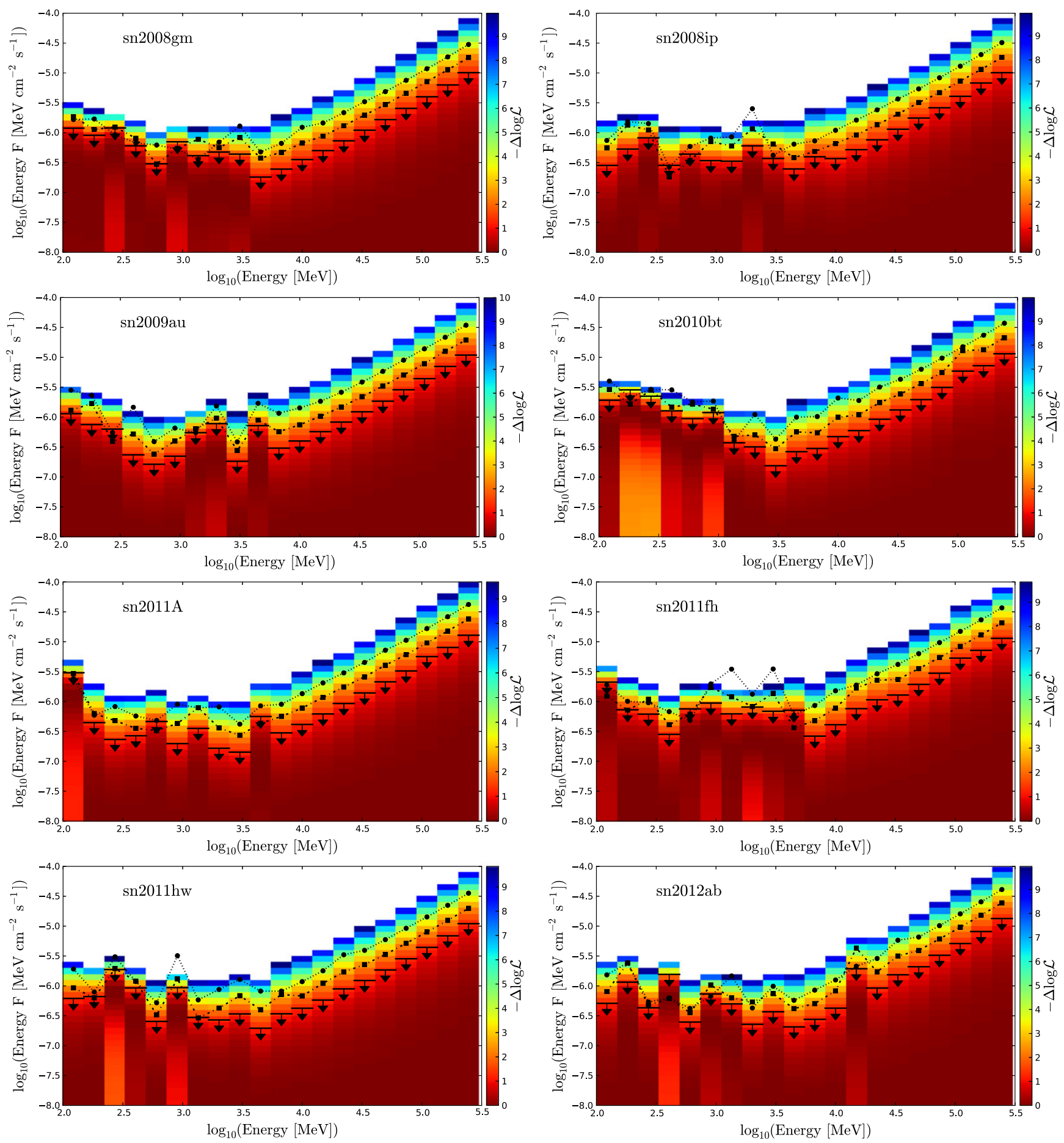

Figure 11. Similar to Figure 4. Colors represent the likelihood profile for each energy for $\Delta T=1$ year. The black arrows indicate the $95 \%$ CL upper limits for $\Delta T=1$ year, while the dotted-dashed and dotted lines represent the $95 \%$ CL upper limit for $\Delta T=6$ months and $\Delta T=3$ months, respectively.

windows $\Delta T=1$ year, $\Delta T=6$ months, and $\Delta T=3$ months for all $\mathrm{SNe}$ listed in Table 1.

\section{REFERENCES}

Abdo, A., Ackermann, M., Ajello, M., et al. 2010, Sci, 329, 817 Abdo, A. A., Ackermann, M., Ajello, M., et al. 2009, ApJS, 183, 46 Ackermann, M., Albert, A., Anderson, B., et al. 2014, PhRvD, 89, 042001 Ade, P. A. R., Aghanim, N., Alves, M. I. R., et al. 2014, A\&A, 571, A1 Atwood, W. B., Abdo, A. A., Ackermann, M., et al. 2009, ApJ, 697, 1071 Bartlett, M. 1953, Biometrika, 40, 306
Bregeon, J., Charles, E., \& Wood, M. 2013, arXiv:1304.5456

Cheung, C. C., Jean, P., Shore, S., et al. 2013, ATel, 5649, 1C Chevalier, R. A., \& Irwin, C. M. 2011, ApJL, 729, L6

Cooke, J., Sullivan, M., Barton, E. J., et al. 2009, Natur, 460, 237 Fransson, C., Ergon, M., Challis, P. J., et al. 2014, ApJ, 797, 118 Hays, E., Cheung, T., \& Ciprini, S. 2013, ATel, 5302, 1H Hill, A., Cheung, C., \& Jean, P. 2013, e-print (arXiv:1308.6281) Katz, B., Sapir, N., \& Waxman, E. 2011, arXiv:1106.1898

Kelner, S. R., Aharonian, F. A., \& Bugayov, V. V. 2006, PhRvD, 74, 034018 Law, N. M., Kulkarni, S. R., Dekany, R. G., et al. 2009, PASP, 121, 1395 Margutti, R., Milisavljevic, D., Soderberg, A. M., et al. 2014, ApJ, 780, 21 Murase, K., Thompson, T., Lacki, B., et al. 2011, PhRvD, 84, 043003 
Murase, K., Thompson, T., \& Ofek, E. 2014, MNRAS, 440, 2528

Neyman, J., \& Pearson, E. S. 1928, Biometrika, 20A, 175

Nolan, P. L., Abdo, A. A., Ackermann, M., et al. 2012, ApJS, 199, 31

Ofek, E. O., Cameron, P. B., Kasliwal, M. M., et al. 2007, ApJL, 659, L13

Ofek, E. O., Lin, L., Kouveliotou, C., et al. 2013, ApJ, 768, 47

Ofek, E. O., Rabinak, I., Neill, J. D., et al. 2010, ApJ, 724, 1396

Ofek, E. O., Sullivan, M., Shaviv, N. J., et al. 2014a, ApJ, 789, 104

Ofek, E. O., Zoglauer, A., Boggs, S. E., et al. 2014b, ApJ, 781, 42
Quimby, R. M., Kulkarni, S. R., Kasliwal, M. M., et al. 2011, Natur, 474, 487

Rau, A., Kulkarni, S. R., Law, N. M., et al. 2009, PASP, 121, 1334

Rolke, W. A., López, A. M., \& Conrad, J. 2005, NIMPA, 551, 493

Smith, N., Hinkle, K. H., \& Ryde, N. 2009, AJ, 137, 3558

Sveshnikova, L. G. 2003, A\&A, 409, 799

Svirski, G., Nakar, E., \& Sari, R. 2012, ApJ, 759, 108

Yaron, O., \& Gal-Yam, A. 2012, PASP, 124, 668

Zhang, T., Wang, X., Wu, C., et al. 2012, AJ, 144, 131 